\title{
Behavioral and nociceptor states of inflammatory pain across timescales in $2 \mathrm{D}$ and $3 \mathrm{D}$
}

Manon Bohic ${ }^{1,2, \dagger}$, Luke A. Pattison ${ }^{3, \dagger}$, Z. Anissa Jhumka ${ }^{4,5}$, Heather Rossi ${ }^{4,5}$, Joshua K. Thackray ${ }^{7,8}$, Matthew Ricci ${ }^{9,10}$, William Foster ${ }^{4,5}$, Justin Arnold ${ }^{4,5}$, Nahom Mossazghi ${ }^{12}$, Eric A. Yttri ${ }^{11,12}$, Max A. Tischfield ${ }^{1,6,8}$, Ewan St. John Smith ${ }^{3}$, Ishmail Abdus-Saboor ${ }^{4,5,{ }^{*}}$, Victoria E. Abraira ${ }^{1,2, *}$

${ }^{1}$ Cell Biology and Neuroscience Department, Rutgers University, The State University of New Jersey, New Brunswick, United States of America

${ }^{2}$ W.M. Keck Center for Collaborative Neuroscience, Rutgers University, The State University of New Jersey, New Brunswick, United States of America

${ }^{3}$ Department of Pharmacology, University of Cambridge, Cambridge, United Kingdom

${ }^{4}$ Zuckerman Mind Brain Behavior Institute and Department of Biological Sciences, Columbia University, New York, New York, United States of America

${ }^{5}$ Department of Biology, University of Pennsylvania, Philadelphia, Pennsylvania, United States of America

${ }^{6}$ Child Health Institute of New Jersey, Robert Wood Johnson Medical School

${ }^{7}$ Human Genetics Institute of New Jersey, Rutgers, The State University of New Jersey, Piscataway, NJ, United States of America

${ }^{8}$ Tourette International Collaborative Genetics Study (TIC Genetics)

${ }^{9}$ Data Science Initiative, Brown University, Providence, Rhode Island, United States

${ }^{10}$ School of Computer Science and Engineering, The Hebrew University of Jerusalem, Jerusalem, Israel

${ }^{11}$ Department of Biomedical Engineering, Carnegie Mellon University, Pittsburgh, PA, United States of America

${ }^{12}$ Department of Biological Sciences, Carnegie Mellon University, Pittsburgh, PA, United States of America

$\dagger$ These authors contributed equally

* Corresponding authors: victoria.abraira@rutgers.edu, ia2458@columbia.edu

Keywords Inflammation, Pain, Carrageenan, Behaviour, Nociception, Electrophysiology

\section{Highlights}

- Automated identification of millisecond-resolved behaviors that separate inflammation-induced mechanical allodynia versus hyperalgesia.

- Sensory neurons innervating the inflamed paw show heightened excitability and nociceptive channel activity.

- Automated identification of spontaneous inflammation-induced behaviors uncovered by computer vision/machine learning during exploratory behavior in an open arena.

- Reduction in evoked pain signatures by an anti-inflammatory drug does not equal return to preinjury state. 


\section{Abstract}

Inflammatory pain represents a complex state involving sensitization of peripheral and central neuronal signalling. Resolving this high-dimensional interplay at the cellular and behavioral level is key to effective therapeutic development. Here, using the carrageenan model of local inflammation of the hind paw, we determine how carrageenan alters both the physiological state of sensory neurons and behaviors at rapid and continuous timescales. We identify higher excitability of sensory neurons innervating the site of inflammation by profiling their physiological state at different time points. To identify millisecond-resolved sensory-reflexive signatures evoked by inflammatory pain, we used a combination of supervised and unsupervised algorithms, and uncovered abnormal paw placement as a defining behavioral feature. For long-term detection and characterization of spontaneous behavioral signatures representative of affective-motivational pain states, we use computer vision coupled to unsupervised machine learning in an open arena. Using the non-steroidal antiinflammatory drug meloxicam to characterize analgesic states during rapid and ongoing timescales, we identify a return to pre-injury states of some sensory-reflexive behaviors, but by and large, many spontaneous, affective-motivational pain behaviors remain unaffected. Taken together, this comprehensive exploration across cellular and behavioral dimensions reveals peripheral versus centrally mediated pain signatures that define the inflamed state, providing a framework for scaling the pain experience at unprecedented resolution.

\section{Introduction}

Pain driven by insults generating strong inflammatory responses represents a major unmet health burden. Heightened nociception during periods of inflammatory pain is protective, serving to promote repair and recovery (Smith and Lewin, 2009). However, during chronic inflammatory diseases such as rheumatoid arthritis or osteoarthritis, prolonged periods of heightened nociception do not confer biological advantages, but rather unmitigated suffering. Despite this, approved therapeutic options for treating the pain associated with inflammation are often inadequate or accompanied by undesirable side effects (Ghouri and Conaghan, 2019; Woodcock, 2009). Development of novel therapeutics will rely on a greater understanding of the complexities underlying pain, from cellular and molecular changes within the nociceptive system to the behavioral level. Preclinical rodent models of inflammatory pain provide an entry point to address this problem. In attempting to discover genes and neural circuits that drive inflammatory pain in mice, it is necessary to investigate the dynamic interplay from nociceptor sensitization, through to the highly nuanced behaviors that result from altered neural activity, and this should be conducted in a cohesive experimental framework.
The physiological state of the nociceptor is a cardinal driver of inflammatory pain, with numerous studies demonstrating greater excitability of sensory neurons following exposure to inflammatory mediators (Davidson et al. 2014). The use of retrograde tracers permits identification of the neurons innervating a particular area of the body, thus enabling greater resolution of the functional and expression changes induced with inflammation by focusing on the cells projecting to the afflicted area (Chakrabarti et al. 2018). In terms of behavior, traditional methods for studying the pain status of rodents often rely on evoked pain measures, whereby the degree of pain experienced by subjects is inferred from their latency to respond to the application of a noxious stimulus (Barrot 2012). Such approaches have been criticized for not fully capturing the nature of pain in humans and their poor correlation with how pain is assessed in the clinic (Mao 2012). Thus, more objective and comprehensive tools to study animal behavior are necessary. Recent advances in computer vision and machine learning have led to the development of more unbiased techniques to monitor evoked animal response such as PAWS (Pain Assessment at Withdrawal Speed) (Jones et al. 2020) and BSOiD (Behavioral Segmentation of Open-Field in DeepLabCut). Both techniques utilize high-frame rate videography to detect subtle micro- 
movements animals perform in response to innocuous or noxious stimulation, followed by analysis of rich data sets by supervised or unsupervised machine learning algorithms. MoSeq (Motion Sequencing) represents a complementary technology using a slightly different approach (Wiltschko et al., 2015). 3D vision is applied to observe an animal's spontaneous behavior in its entirety, followed by unsupervised machine learning to extract the dozens of motion sequences or syllables that make up mouse body language. The overall goal of the work described here is to utilize multiple dimensions of analysis - from the physiological state of the sensory neuron to complex behaviors, to uncover novel features that define the inflammatory pain state in mice.

Local application of carrageenan is one of the most widely used models of inflammatory pain in preclinical rodent models. Intraplantar injection of carrageenan into the hind paw induces robust edema within four hours (Morris 2003), whereas heightened sensitivity to both thermal and mechanical stimuli appears to persist for at least a day (Hargreaves et al. 1988; Buisseret et al. 2019). To date, studies characterizing the carrageenan model and assessing the efficacy of therapeutic agents have mostly relied on histological analyses and pain behavioral assays that focus on latency to paw withdrawal or withdrawal threshold (Pritchard et al. 2016; Rock et al. 2018; Buisseret et al. 2019; Miyake et al. 2019). With this rather limited level of characterization of a complex state, what prominent features of inflammatory pain in rodents might have gone undetected? Since pain encompasses molecular, cellular, and highly specific behavioral signatures, or "modules" - to fully understand the inflammatory pain state for assessing novel points for therapeutic intervention, a multidisciplinary approach is needed.

Here, we utilize PAWS, B-SOiD and MoSeq for fine-resolution behavioral mapping alongside whole-cell electrophysiology recordings and immunohistochemistry analyses of sensory neurons innervating the site of inflammation. We determined that retrograde labeled sensory neurons innervating the hind paw show decreased action potential threshold and greater magnitude voltage-gated inward currents in the hours immediately following carrageenan-induced inflammation. In terms of heightened sensitivity to mechanical stimuli during inflammation just seconds after stimulus delivery, we identify increased paw guarding as a prominent feature, with unique paw guarding behaviors for application of innocuous versus noxious stimuli. Through computer vision coupled to machine learning, we uncover sets of spontaneous behavioral syllables representative of pain and pain relief states in freely moving mice, as well as behavioral modulation with the analgesic drug meloxicam at baseline, in the absence of injury. Lastly, we demonstrate that analgesic pain relief as revealed by rapid paw movements captures only a subset of pain state, and may not represent a true return to basal conditions. This surprising result has major implications for testing the clinical efficacy of new analgesics when only using traditional paw withdrawal approaches to measure pain.

\section{Results}

\section{Carrageenan induces transient changes in paw swelling and weight bearing but evokes prolonged thermal hyperalgesia}

Carrageenan-induced inflammation has been widely studied by neuroscientists and immunologists, usually coupled with evoked pain behaviors and/or gait analysis to identify new analgesic targets. Indeed, such approaches, as summarized in Figure 1A, have demonstrated that independent administration of prostaglandin D2glycerol, cannabinoids and ivabradine all alleviate evoked pain behaviors in rodents following induction of inflammation with carrageenan (Buisseret et al., 2019; Miyake et al., 2019; Rock et al., 2018). However, since many patients still have poorly treated pain driven by inflammation, this suggests that additional signatures need to be identified in rodent models that might serve as new analgesic targets. As a starting point in our studies, we began by focusing on traditional metrics of measuring carrageenan-induced inflammation, for future comparison to the new experimental pipeline developed here. 


\section{Traditional approaches for measuring inflammatory pain}

\section{A}
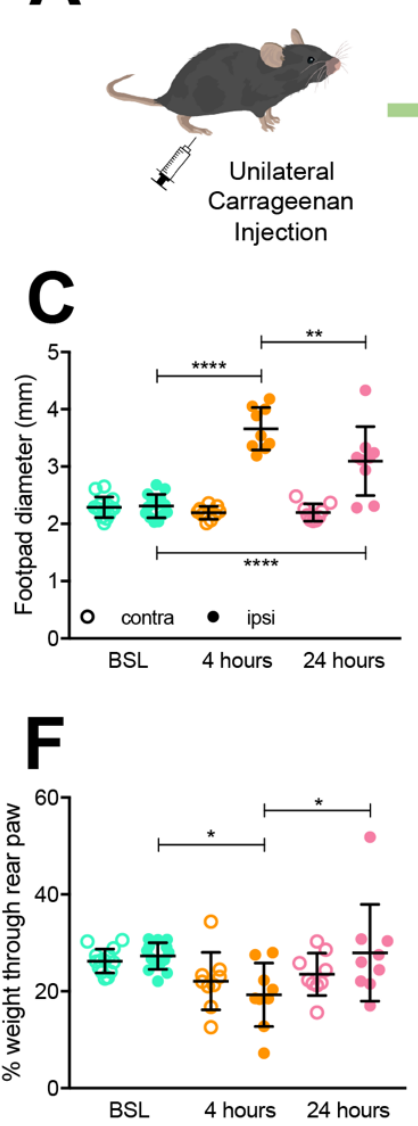



D

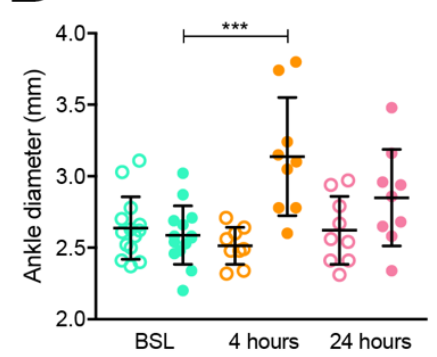

G

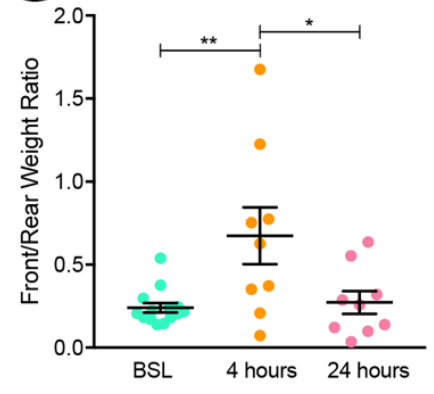

B

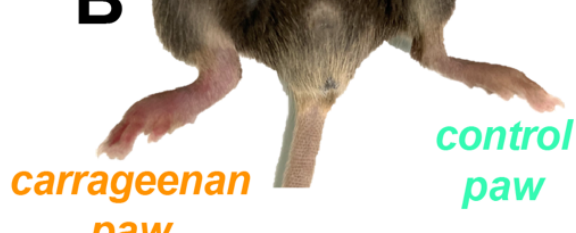

paw
E Hargreaves thermal

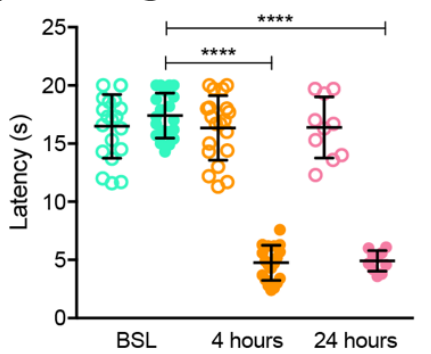

H

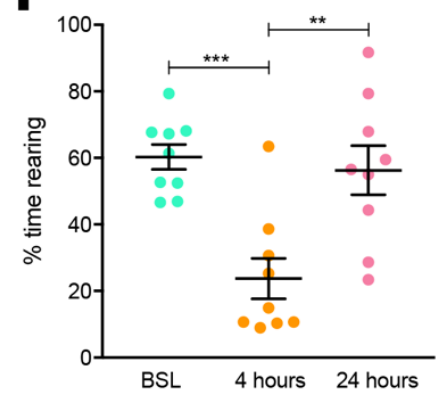

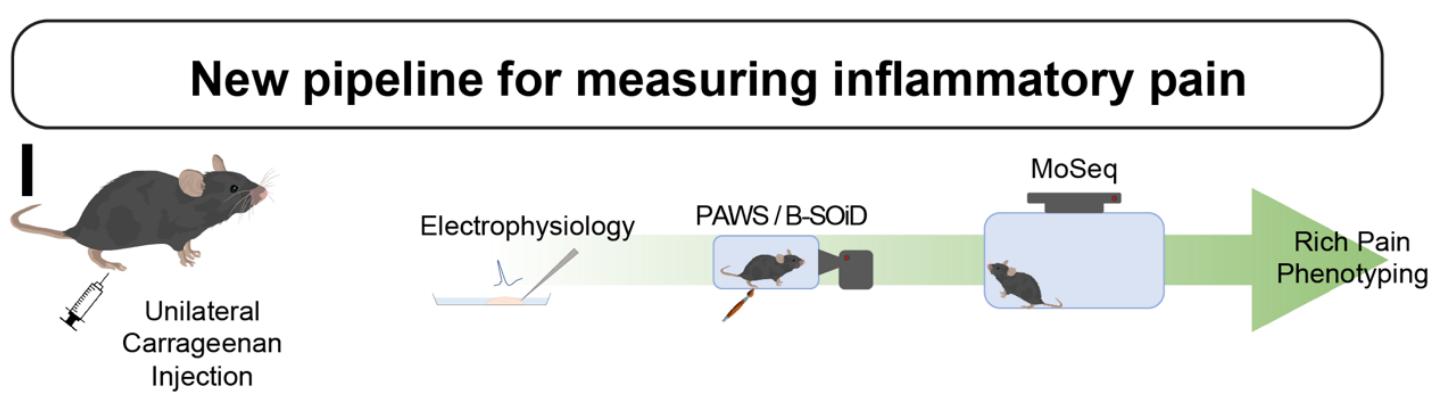

Figure 1. Unilateral injection of carrageenan into the hind paw of mice results in swelling and weight bearing deficits. (A) Schematic representation of a traditional experimental workflow to assess the pain-status of rodents. (B) Noticeable inflammation of the injected (ispi) hind paw was observed 4-hours post-injection with carrageenan compared to the non-injected hind paw (contra). Swelling of both the (C) footpad and (D) ankle was quantified for both hind paws with digital calipers before, 4-hours-post and 24-hours-post induction of inflammation with carrageenan. (E) Hargreaves measurement of carrageenan-induced heat hypersensitivity at baseline, and following carrageenan injection at 4-hours and 24-hours. (F) The weight through each hind paw, $(\mathbf{G})$ ratio of weight through front paws compared to the rear and $\mathbf{( H )}$ time spent rearing by mice was assessed with dynamic weight bearing apparatus. (I) Combining electrophysiology with the advanced behavioral assays PAWS, B-SOiD and MoSeq allows for high resolution examination of the biobehavioral status of animals. Data are presented as mean \pm standard deviation. ${ }^{*} \mathrm{p}<$ $0.05, * * \mathrm{p}<0.01, * * * \mathrm{p}<0.001, * * * * \mathrm{p}<0.0001$, one-way ANOVA followed by Bonferroni post-hoc.

Following unilateral injection of carrageenan into the hind paw, we first sought to expand assessment of mouse behavior following unilateral injection of carrageenan into the hind paw using dynamic weight-bearing. As expected, we observed noticeable swelling of both the footpad (precarrageenan, $2.31 \pm 0.20 \mathrm{~mm}$, 4-hours, $3.66 \pm 0.37$ $\mathrm{mm}, \mathrm{p}<0.0001$, ANOVA with Bonferroni posthoc, Figure 1B-C) and ankle (pre-carrageenan, 2.59 $\pm 0.20 \mathrm{~mm}$, 4-hours, $3.14 \pm 0.41 \mathrm{~mm}, \mathrm{p}<0.001$, ANOVA with Bonferroni post-hoc, Figure 1B,D) 
of the injected hind paw 4 hours after the induction of inflammation. No such swelling was observed for the contralateral hind paw (footpad precarrageenan, $2.29 \pm 0.18 \mathrm{~mm}$, footpad 4-hours, 2.19 $\pm 0.11 \mathrm{~mm}$, ankle pre-carrageenan, $2.64 \pm 0.22$ $\mathrm{mm}$, ankle 4-hours, $2.51 \pm 0.13 \mathrm{~mm}$, Figure 1B-D). Swelling of the injected footpad persisted 24 hours (pre-carrageenan, $2.31 \pm 0.20 \mathrm{~mm}, 24$-hours, 3.10 $\pm 0.60 \mathrm{~mm}, \mathrm{p}<0.0001$, ANOVA with Bonferroni post-hoc, Figure 1C), while ankle inflammation had mostly resolved by this time (pre-carrageenan, $2.59 \pm 0.20 \mathrm{~mm}, 24$-hours, $2.85 \pm 0.34 \mathrm{~mm}$, Figure 1D).

Thermal hypersensitivity, a hallmark of inflammatory pain, was assessed at baseline, 4hours post-carrageenan injection, considered to be an acute pain state and 24-hours post-carrageenan when mice traditionally exhibit peak inflammation-induced hypersensitivity. Hargreaves measurement of heat hypersensitivity shows that mice develop acute (4-hours post-injection) and longer-lasting (24-hours post-injection) heat hypersensitivity of the carrageenan injected paw compared to the contralateral paw (Figure 1E). Although heat sensitivity thresholds are significantly altered at both time points compared to baseline sensitivity (pre-carrageenan, $17.4 \pm$ $0.43 \mathrm{~s}, 4$-hours, $4.76 \pm 0.34 \mathrm{~s}, 24$-hours, $4.92 \pm 0.28$ $\mathrm{s}, \mathrm{p}<0.0001$, ANOVA with Bonferroni post-hoc, Figure 1E), Hargreaves is not sensitive enough to distinguish between acute and longer-lasting pain at 4-hours and 24-hours post injection (4-hours, $4.76 \pm 0.34 \mathrm{~s}, 24$-hours, $4.92 \pm 0.28 \mathrm{~s}$, Figure 1E). We next sought to expand assessment of mouse behavior using dynamic weight-bearing. The observed swelling corresponded to reduced weight

\section{Inflammatory pain sensory-evoked behavioral modules uncovered at millisecond resolution with a supervised approach}

To determine sensitivity to mechanical stimuli during heightened pain states, traditional approaches use application of von Frey hair filaments and record the animal's response or lack thereof. In such cases, increased or decreased mechanical sensitivity can be observed but loading through the rear paw for the injected side after 4-hours (pre-inflammation, $27.26 \pm 2.74 \%$, 4hours, $19.27 \pm 6.55 \%, \mathrm{p}<0.05$, ANOVA with Bonferroni post-hoc, Figure 1F), but not 24-hours after carrageenan injection (pre-inflammation, $27.26 \pm 2.74 \%, 24$-hours, $27.94 \pm 9.97 \%$, Figure $1 \mathrm{~F})$. This finding coincided with mice placing more weight through their front paws relative to their rear paws at the 4-hour time-point (preinflammation, $0.24 \pm 0.11$, 4-hours, $0.67 \pm 0.51$, p $<0.01$, ANOVA with Bonferroni post-hoc, Figure $1 \mathrm{G}$ ), the distribution of weight-bearing more closely resembled pre-inflammation levels after 24hours of inflammation (pre-inflammation, $0.24 \pm$ 0.11 , 24-hours, $0.27 \pm 0.21$, Figure 1G). The proportion of time animals spent rearing, an explorative stance relying on significant weightbearing through the rear paws, was reduced 4hours after injection of carrageenan (preinflammation, $60.27 \pm 11.26 \%$, 4-hours, $23.73 \pm$ $18.25 \%, \mathrm{p}<0.001$, ANOVA with Bonferroni posthoc, Figure 1H). Again, the time animals spent rearing 24-hours after induction of inflammation was more comparable to pre-inflammation measures (pre-inflammation $60.27 \pm 11.26 \%, 24$ hours, $56.27 \pm 22.12 \%$, Figure $1 \mathrm{H}$ ). These findings build upon previous reports of mechanical and thermal allodynia following induction of inflammation with carrageenan, demonstrating a weight-bearing deficit that correlates with the peak of physical inflammation and longer-lasting thermal hypersensitivity. Having expanded basic understanding of carrageenan-induced inflammation, we next sought to identify new biobehavioral signatures in the carrageenan model using our refined pipeline summarized in Figure 1I.

mechanical allodynia and/or hyperalgesia, whether innocuous stimulation becomes painful or noxious stimulation becomes even more painful, respectively, are very difficult to unambiguously disentangle. Moreover, other important behavioral features of heightened pain states may go unnoticed with a sole focus on paw withdrawal frequency or threshold. To provide added resolution, we recently developed an open-source automated pain scale with custom software called PAWS (Pain Assessment at Withdrawal Speeds) 
bioRxiv preprint doi: https://doi.org/10.1101/2021.06.16.448689- this version posted June 17, 2021. The copyright holder for this preprint (which was not certified by peer review) is the author/funder, who has granted bioRxiv a license to display the preprint in perpetuity. It is made available under aCC-BY 4.0 International license.

that takes high-frame rate behavioral data to quantify rapid reflexive behaviors and defensive coping behaviors like paw guarding and shaking (Jones et al., 2020). Here, we deployed PAWS in the carrageenan inflammatory model, seeking to uncover behavioral modules that may have gone undetected with traditional approaches.
We followed the same experimental timeline described above and recorded animal behavior at 2,000 frames per second (fps) with a focus on the carrageenan injected paw at baseline, as well as 4and 24-hours after paw injection. We used the ProAnalyst program for paw tracking, followed by analyzing the videos using the PAWS software

\section{High-speed videography + automated paw tracking}


PAWS behavioral features with carrageenan inflammation model
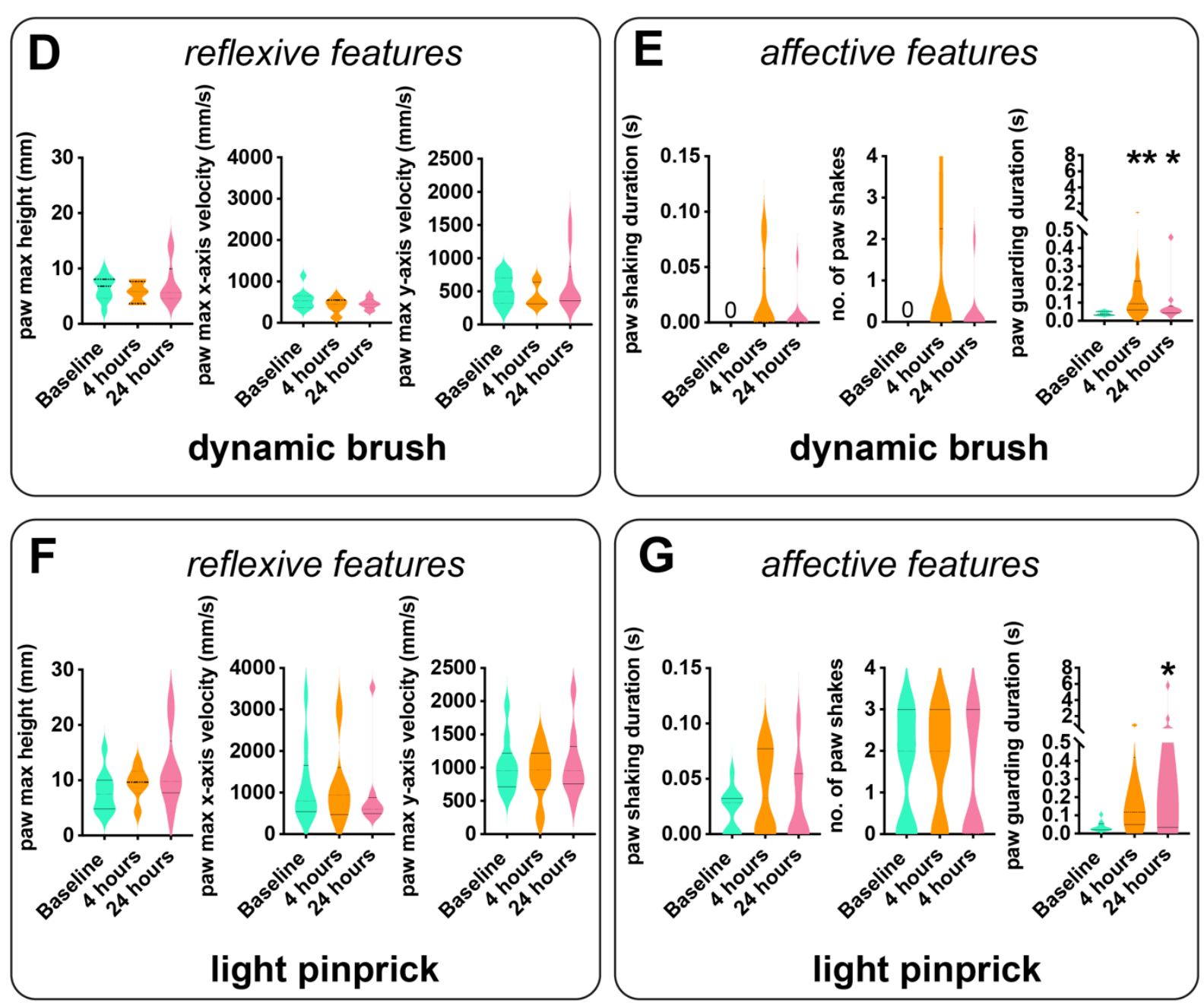
Figure 2. PAWS automated pain assessment platform detects increase in defensive coping behaviors following carrageenan injection. A) A behavioral response to a somatosensory stimulus at baseline. (B) Post-carrageenan injection mice guard the paw in the air for extended time. $(\mathrm{A}, \mathrm{B})$ Green lines show paw trajectory pattern across entire behavior, and mouse image shows single frame with paw at its apex. (C) Image drawn in Biorender and modified from Jones et al., 2020. PAWS software measures paw height, velocity, shaking, and guarding in $\mathrm{X}$ and $\mathrm{Y}$ axes. Reflexive behaviors occur before paw reaches its apex ( $\left.\mathrm{t}^{*}\right)$, whereas affective behaviors occur after $t^{*}$. (D,E) No behavioral changes detected in reflexive features with dynamic brush comparing baseline to 4 and 24hour post-carrageenan injection, whereas affective features such as paw guarding are upregulated. (F,G) No behavioral changes detected in reflexive features with light pinprick comparing baseline to 4 and 24hour post-carrageenan injection, whereas affective features such as paw guarding are upregulated. $\mathrm{N}=10$ mice per group and Wilcoxon matched-pairs sign rank test was performed to determine statistical significance. ${ }^{*} \mathrm{p}<0.05,{ }^{*} * \mathrm{p}<0.01$, *** $\mathrm{p}<0.001$, **** $\mathrm{p}<0.0001$

(Fig. 2A-C). Using the dynamic brush stimulus that predominantly activates large diameter mechanoreceptors (Abdus-Saboor et al., 2019), we do not observe any significant differences across timepoints in reflexive behavioral features that occur just after stimulus onset (Fig. 2D). However, coping behaviors, such as paw shaking and paw guarding, more commonly associated with affect, do show an increase after carrageenan injection, most notably paw guarding behavior (Fig. 2E). With the application of the mildly noxious light pinprick stimulus, which chiefly activates nociceptors (Abdus-Saboor et al., 2019), we observe an identical pattern to that observed with the dynamic brush. Reflexive behavioral features remain unchanged after carrageenan injection, whereas the affective behavioral features, namely paw guarding is upregulated (Fig. 2F). Moreover, we find that according to the type of stimulus, the elicited-guarding duration occurs in an intrinsic

\section{Unsupervised detection of rapid pain behavior signatures during inflammation}

The PAWS platform takes high frame rate videography data and measures pre-defined intuitive features that have meaning to pain researchers (Jones et al., 2020). However, top-down approaches may perpetuate a user bias. Moreover, there may be paw dynamic features during inflammatory pain, discoverable by unsupervised approaches, that may reside below the level of easy experimenter detection. To address this, we employed B-SOiD, an unsupervised machine learning algorithm that parses spatiotemporal patterns from position data (Hsu and Yttri, 2021). For accurate comparison, we used the same highspeed behavioral data collected in Fig. 2. As inputs, time-specific fashion. Indeed, while paw guarding duration evoked by dynamic brush is significantly increased as early as 4-hours post-injection (Wilcoxon matched-pairs signed rank test: 4-hours vs baseline $\mathrm{p}<0.05$ 0.020; 24-hours versus baseline $\mathrm{p}<0.05$ ), paw guarding duration evoked by light pin prick is singularly upregulated at 24-hours post-inflammation (Wilcoxon matched-pairs signed rank test: 4 -hours versus baseline $\mathrm{p}=\mathrm{ns} ; 24 \mathrm{~h}$ versus baseline $\mathrm{p}<0.05)$. Taken together, these data demonstrate in an unbiased and automated fashion, that carrageenan-induced sensitization does not alter the speed or height in which an animal retracts its paw to mechanical stimuli. Rather, this immunogenic compound specifically alters the responses to mechanical stimuli such that defensive coping behaviors are more frequent. These results suggest that the neural circuits that drive paw guarding are specifically sensitized by carrageenan.

we used two positions within the hind paw and two reference points as identified with the deep neural network DeepLabCut (Fig. 3A, see Methods). B$\mathrm{SOiD}$ then identified and extracted unique clusters constituting conserved motor responses to these stimuli (Fig. 3B; categorical names were assigned to clusters after the fact). Importantly, the behaviors were identified without any knowledge beyond point position, and the B-SOiD model developers were blinded to stimulus identity and treatment group. Eleven sub-clusters were found in both stimulation contexts, and we observed changes in response to stimuli beginning at 4-hours post-injection (Fig 3C-G). For example, carrageenan injection led to increased guarding across stimuli as well as a lift-to-hover pattern that was quantified as a distinct behavioral module 
bioRxiv preprint doi: https://doi.org/10.1101/2021.06.16.448689. this version posted June 17, 2021. The copyright holder for this preprint (which was not certified by peer review) is the author/funder, who has granted bioRxiv a license to display the preprint in perpetuity. It is made available under aCC-BY 4.0 International license.

from guarding. The hovering pattern can be observed in traces of a representative video where the stimulated paw height is above zero (red line), while the speed is at zero (black line) (Figure 3C right panels), and when repeated, appears analogous to shaking.

These behaviors and distributions are similar to those observed by human raters and top-down supervised approaches - though differ in some important aspects. The automated behavioral assessment extracted two guarding types specific to the foot stimulus presented (i.e. angled versus flat guard). These phenotypes were present across animals and both the 4- and 24-hour time points and were distinct from each other in both height and foot posture. Since the "angled guard" (paw lifted and perpendicular to the surface) was upregulated with the innocuous stimulus, this might be a behavior specific to touch neurons and thus mechanical allodynia. Conversely, the "flat guard" was upregulated with the noxious stimulus (paw lifted and parallel to the surface), and might be associated specifically with nociceptors and mechanical hyperalgesia. To date, there are no behavioral signatures beyond the discovery here

\section{B-SOiD Unsupervised Algorithm for Detecting Sub-second Behaviors}



single trial examples; frames (aligned to rest before paw lift or hovering)

$\mathbf{E}$ ang7!ed par g]uard

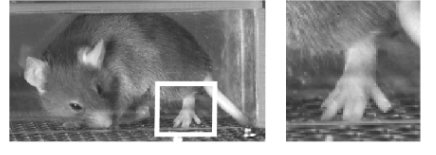

"allodynia” behavior

flat paw guard
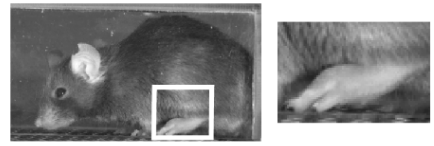

"hyperalgesia” behavior
E

dynamic brush



bsl $4 \mathrm{hr} 24 \mathrm{hr}$
G light pinprick


bsl $4 \mathrm{hr} 24 \mathrm{hr}$ composite data ( $n=10$ mice/time point) 
Figure 3. Unsupervised discovery of inflammatory pain behavioral responses to foot stimulation. A) Identification of inputs to be used for unsupervised classification in B-SOiD. (B) Low-dimensional projection of identified feature clusters. Colors of sub-clusters indicate their behavioral group assignment (red=at rest, green=lift, brown=hover, yellow=angled guard, blue=flat guard). (C) Representative responses to dynamic brush and (D) light pinprick at baseline, 4-hour, and 24-hour timepoints post-carrageenan injection. Responses are color-coded by the identified action type in panel (B), with speed (green) and height (black) on the left (both in arbitrary units). (E) Examples of the two forms of guarding identified by B-SOiD, which may be indicative of the activation of different subsets of sensory neurons (mechanoreceptors by brush, inducing angled guard, nociceptors by pinprick, inducing flat guard). (F) Distribution of behaviors observed across dynamic brush trials. (G) Distribution of behaviors observed across light pinprick trials. For F and G, sampling window is $-25 \mathrm{~ms}$ to $500 \mathrm{~ms}$ relative to movement onset.

that allows researchers to distinguish mechanical allodynia versus mechanical hyperalgesia. Taken together, an unbiased and agnostic algorithm can extract reliable behavioral modules generated by

\section{MoSeq detects spontaneous behavioral signatures of carrageenan-induced inflammation}

Although evoked behavioral measurements provide one critical domain of the pain neuroaxis, spontaneous bouts of pain are what chronic pain patients experience most frequently (Ott and Maihöfner, 2018). To date, the majority of inflammatory pain behavioral measurements in rodents have only proxied evoked measurements, which greatly limits the translational impact of our rodent models. Understanding how molecules and circuits influence pain requires the ability to objectively organize information about similarities and differences within complex pain behavior datasets. Recent advances in computer vision and machine learning allow us to do this more efficiently. Using the previously described MoSeq technology (Wiltschko et al., 2015; 2020), we explored whether carrageenan-induced inflammatory pain affects spontaneous animal behavior in a meaningful way that can be detected by MoSeq. Singular measurements such as paw withdrawal latency to a stimulus, including the Hargreaves thermal assay, do not provide adequate resolution to separate potentially disparate behavioral states. Conversely, unsupervised analysis of micro-movements performed by freelymoving mice filmed in 3D from above (figure 4B) provides a rich dataset and an unbiased approach to the study of mouse spontaneous behavior. Here, we find that MoSeq automatically detects as many as 62 unique syllables or modules, the large inflammatory pain, and moreover, it can provide valuable information about sensitization of the underlying neural circuits.

majority of which were differentially utilized by animals at baseline compared to 4-hours and 24hours after carrageenan injection (heatmap Figure $4 \mathrm{C})$. We classified these 62 syllables as representing one of four types of behavior: exploratory/escape behavior, grooming, pause and rearing. While exploratory behavior is mainly unaffected, probably on account of the curious nature of mice, grooming and rearing behavior is strongly downregulated because of pain. For example, we found that rearing behavior (syllable 42, Figure $4 \mathrm{D}, \quad \mathrm{E})$ is affected by carrageenan-induced inflammatory pain at 4-hours and 24-hours postinjection (Figure 4D). This result suggests that this form of rearing behavior may cause heightened mechanical allodynia to animals and is therefore inhibited by inflammation. Of the 27 subtypes of pausing detected by MoSeq, about half are affected by inflammatory pain, either up or downregulated. Thus, inflammatory pain vastly alters the spontaneous display of behavior. Further linear discriminant analysis (LDA) of transition probabilities across time and pain states shows that MoSeq can detect an important behavioral transition in spontaneous behavior induced by inflammatory pain between the 4-hours and 24hour time points (Figure 4F), which was undetected by Hargreaves (Figure 1E shows that Hargreaves cannot separate inflammatory pain behavior at 4 versus $24 \mathrm{hrs}$ ). The detection of this evolution in the pain state of the animal could have important implications as to the different neuronal networks involved at these two time points (peripheral versus more central) and prove crucial 
bioRxiv preprint doi: https://doi.org/10.1101/2021.06.16.448689. this version posted June 17, 2021. The copyright holder for this preprint (which was not certified by peer review) is the author/funder, who has granted bioRxiv a license to display the preprint in perpetuity. It is made available under aCC-BY 4.0 International license.

to efficient anti-inflammatory drug development. The mosaic of behavioral changes induced by inflammatory pain goes to show the complexity of mouse behavior and the need for unbiased approaches, like MoSeq and subsequent unsupervised analysis of the rich dataset it generates, to fully grasp what spontaneous and ongoing pain is made of.

\section{MoSeq detects spontaneous behavioral modules of inflammatory pain}
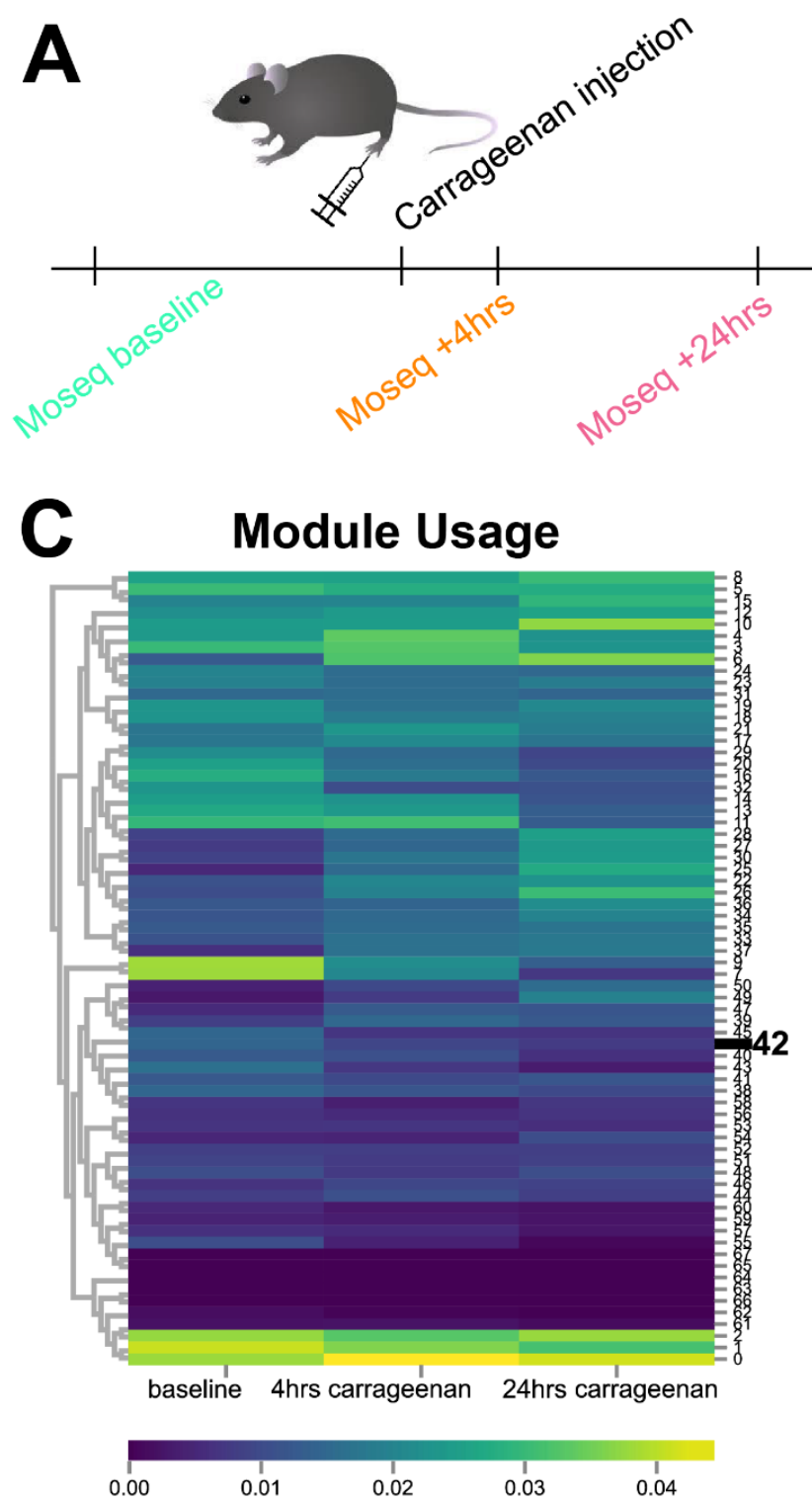

\section{E Module No. 42 Spinogram}

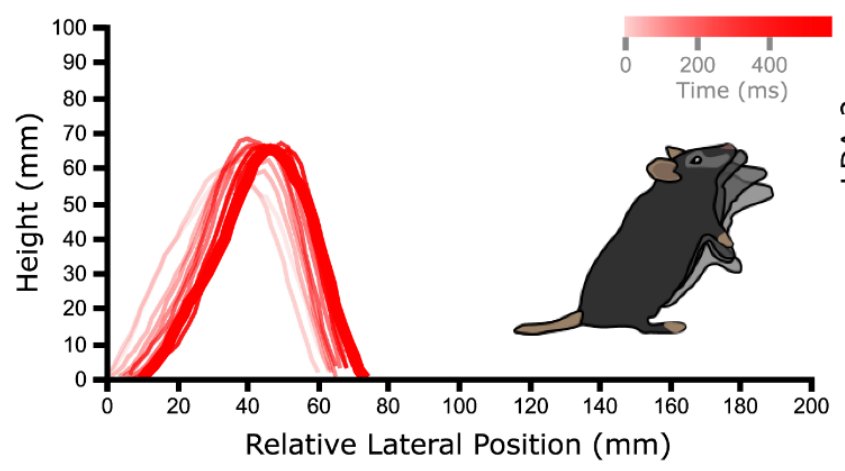

B

Figure 4. MoSeq detects behavioral signatures of carrageenan-induced inflammatory pain (A) Timeline of the experiment. (B) Top-down view of video recording mouse to detect spontaneous behavoirs during open arena exploration (C) Heatmap representation of syllable usage by the three experimental animal groups baseline, 4-hours and 24-hours carrageenan. (D) Example of a module with varying usage at baseline, and following carrageenan injection at 4-hours, 24-hours. (E) Spinogram and mouse representation of syllable 42 (rearing). (F) Representation of linear discriminant analysis (LDA) of MoSeq spontaneous behavior at baseline, and following carrageenan injection at 4-hours, 24-hours.

\section{Electrophysiological characterization of hind paw innervating sensory neurons reveals greatest excitability just hours following carrageenan injection}

Having identified novel behavioral signatures of inflammatory pain at defined time-points, we next sought to determine if activity of site-relevant sensory neurons might correlate with some of the identified behaviors. Sensory neurons innervating the hind paw were identified with the retrograde tracer Fast Blue (Figure 5A) and of acutely cultured lumbar DRG neurons (L2-L5) $\sim 4.46 \%$ were found to be Fast Blue positive (Figure 5B inset). The relative size distribution of the Fast Blue positive population closely resembled that of the total lumbar DRG (Figure 5B), that is to say the hind paw is likely innervated by the full range of neuronal subtypes. Electrophysiological characterization of the Fast Blue populations cultured from the inflamed side (ipsilateral, ipsi.) and non-injected side (contralateral, contra.) revealed a lower rheobase (current injection necessary for action potential discharge) of ipsilateral neurons compared to contralateral neurons after 4-hours of inflammation suggesting the rapid onset of peripheral sensitization following exposure to carrageenan (contra., $272.1 \pm 48.95$ pA, ipsi., $125.1 \pm 25.36 \mathrm{pA}, \mathrm{p}<0.01$, unpaired ttest, Figure 5C,E-F). No significant difference in rheobase was observed between ipsilateral and contralateral neurons at the 24-hour postinflammation time point (contra., $290.00 \pm 61.61$ pA, ipsi., $201.14 \pm 37.74$ pA, Figure 5C). After determining the rheobase, neurons were stimulated at suprathreshold (2X rheobase), neurons isolated from the ipsilateral side after 4-hours of inflammation had a higher tendency to fire multiple action potentials during this stimulation than those from the non-injected side (contra., 1.87 \pm 0.47 , ipsi., $3.00 \pm 0.42, \mathrm{p}=0.1128$, unpaired $\mathrm{t}-$ test, Figure 5D), no such difference was observed 24-hours post-inflammation (contra., $2.92 \pm 0.58$, ipsi., $2.65 \pm 0.42$ ). The neurons recorded from did not differ in any other intrinsic or active parameter measured for either time point, as summarized in Table 1.

\begin{tabular}{l|c|c|c|c|}
\cline { 2 - 5 } & \multicolumn{4}{|c|}{ Hours post-inflammation } \\
\cline { 2 - 5 } & \multicolumn{2}{|c|}{4 hours } & \multicolumn{2}{c|}{24 hours } \\
\cline { 2 - 5 } & Contra $(\mathrm{n}=20)$ & Ipsi $(\mathrm{n}=39)$ & Contra $(\mathrm{n}=20)$ & Ipsi $(\mathrm{n}=28)$ \\
\hline $\begin{array}{l}\text { Resting membrane potential } \\
(\mathrm{mV})\end{array}$ & $-48.20 \pm 1.49$ & $-46.05 \pm 0.83$ & $-49.00 \pm 1.43$ & $-45.64 \pm 1.40$ \\
\hline Capacitance $(\mathrm{pF})$ & $31.23 \pm 2.69$ & $25.90 \pm 1.73$ & $30.77 \pm 4.44$ & $33.17 \pm 3.03$ \\
\hline Rheobase $(\mathrm{pA})$ & $272.10 \pm 48.95^{\star *}$ & $125.10 \pm 25.36^{* *}$ & $290.00 \pm 61.61$ & $201.40 \pm 37.74$ \\
\hline $\begin{array}{l}\text { Action potential amplitude } \\
(\mathrm{mV})\end{array}$ & $80.33 \pm 3.95$ & $84.58 \pm 2.17$ & $85.45 \pm 3.65$ & $87.11 \pm 2.74$ \\
\hline $\begin{array}{l}\text { Half peak duration (ms) } \\
\text { Afterhyperpolarization }\end{array}$ & $3.04 \pm 0.26$ & $3.50 \pm 0.15$ & $3.87 \pm 0.35$ & $3.37 \pm 0.27$ \\
\hline $\begin{array}{l}\text { Amplitude (mV) } \\
\text { duration (ms) }\end{array}$ & $15.43 \pm 1.30$ & $14.10 \pm 0.76$ & $16.61 \pm 0.71$ & $16.15 \pm 0.84$ \\
\hline $\begin{array}{l}\text { \# Action potentials at } \\
\text { suprathreshold }\end{array}$ & $15.36 \pm 1.55$ & $17.82 \pm 1.55$ & $15.48 \pm 1.17$ & $16.45 \pm 1.19$ \\
\hline
\end{tabular}

Table 1 Intrinsic and active properties of hind paw innervating dorsal root ganglion neurons from the carrageenan injected side (Ipsi) and contralateral (Contra) side. Data are mean \pm SEM. ** $\mathrm{p}<0.01$, unpaired t-test. 
bioRxiv preprint doi: https://doi.org/10.1101/2021.06.16.448689; this version posted June 17, 2021. The copyright holder for this preprint (which was not certified by peer review) is the author/funder, who has granted bioRxiv a license to display the preprint in perpetuity. It is made available under aCC-BY 4.0 International license.

The higher excitability of sensory neurons observed at 4-hours post-injection of carrageenan correlates with the peak of physical inflammation, suggesting that mediators released during the inflammatory process may be responsible for sensitizing the neurons. As inflammation subsides, the levels of inflammatory mediators declines, and this corresponds with the degree of excitability of neurons from the ipsilateral side being more comparable to the contralateral side at the later 24-hour post-inflammation time point. Although the inflammatory peak has passed, changes in the expression of nociceptive channels that were initiated during the height of inflammation might begin to manifest and influence behavior at later time points. We thus assessed expression of TRPV1, an ion channel involved in detection of noxious heat, in whole sections of lumbar DRG (L3 and L4). Greater TRPV1 immunoreactivity was observed for sensory neurons innervating the inflamed hind paw compared to DRG isolated from the contralateral side (contra., $20.01 \pm 1.87 \%$, ipsi., $29.88 \pm 3.80 \%, \mathrm{p}=0.0571$, Mann-Whitney test, Figure 5G-H). This result likely explains our earlier observation of thermal hypersensitivity persisting at the 24-hours post-inflammation time point (Figure 1E). Together, these results suggest an adaptation of the sensory neurons in response to the initial inflammatory insult which renders them more sensitive to noxious stimuli, even after the inflammation has subsided. In addition, since sensory neuron excitability decreases by 24-hours post-inflammation, but pain behavioral signatures persist (Figures 2-4), this suggests that central sensitization might drive pain states beyond the initial few hours.

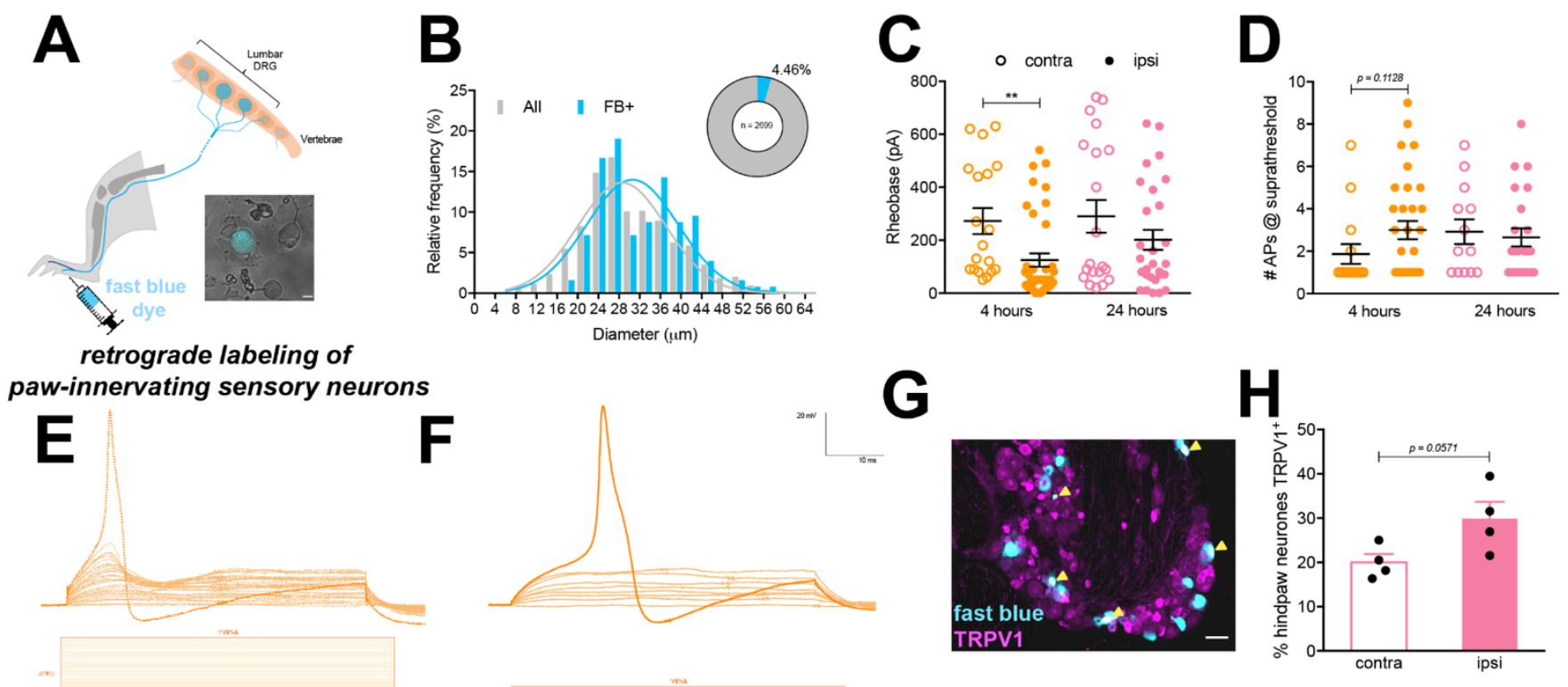

Figure 5. Hind paw innervating sensory neurons cultured from the inflamed side are more excitable than those from the non-injected side during early inflammation. (A) Schematic representation of retrograde labelling of hind paw innervating sensory neurons with Fast Blue, Inset: Fast Blue positive neuron (blue) following acute dissociation, scale $=50 \mu \mathrm{m}$. (B) Relative frequency distributions of dissociated sensory neuron soma diameter from hind paw innervating and total lumbar populations, Insert: Proportion Fast Blue positive cells from acutely dissociated cultures of lumbar DRG. (C) Step-wise current injections were used to determine the rheobase of hind paw innervating sensory neurons from injected (ipsi) and non-injected (contra) sides 4- or 24-hours post-induction of inflammation with carrageenan. (D) Frequency of action potential (AP) discharge following stimulation of sensory neurons with a suprathreshold (2X rheobase). Representative traces from step-wise current injections to determine rheobase of neurons from (E) contralateral and (F) ipsilateral sides 4-hours post injection of carrageenan. (G) A subset of hind paw innervating sensory neurons (blue) express the nociceptive ion channel TRPV1 (magenta), cells positive for both Fast Blue and TRPV1 are identified by yellow pointers, scale $=50 \mu \mathrm{m}$. $(\mathbf{H})$ A higher proportion of hind paw innervating neurons expressed TRPV1 after 24-hours of carrageenan-induced inflammation. ${ }^{* *} \mathrm{p}<0.01$, unpaired t-test.

Next, the activity of voltage-sensitive channels was assessed in voltage-clamp: peak inward and outward currents were measured as the holding voltage was stepped from $-60 \mathrm{mV}$ to $55 \mathrm{mV}$. Inward current was greater for ipsilateral neurons after 4hours of inflammation relative to neurons from the 
bioRxiv preprint doi: https://doi.org/10.1101/2021.06.16.448689; this version posted June 17,2021 . The copyright holder for this preprint (which was not certified by peer review) is the author/funder, who has granted bioRxiv a license to display the preprint in perpetuity. It is made available under aCC-BY 4.0 International license.

non-injected side (peak normalized current: contra., $-0.97 \pm 0.47$, ipsi., $-1.29 \pm 0.54, \mathrm{p}<0.05$, unpaired t-test, Figure 6A-C). The conductance of channels permitting inward current was also found to be higher (peak normalized conductance: contra., $1.00 \pm 0.67$, ipsi., $1.43 \pm 0.74, \mathrm{p}<0.05$, unpaired t-test, Figure 6F-G). However, no difference in the gating properties of channels was observed (Erev: contra., $83.22 \pm 5.45 \mathrm{mV}$, ipsi., $78.14 \pm 3.36 \mathrm{mV}$; Vhalf: contra., $-38.73 \pm 4.13 \mathrm{mV}$, ipsi., $-40.07 \pm 1.40 \mathrm{mV})$. By contrast, peak current, conductance and gating properties were

\section{Increased voltage-gated inward currents after carrageenan}

\section{A \\ 4 hours}

24 hours
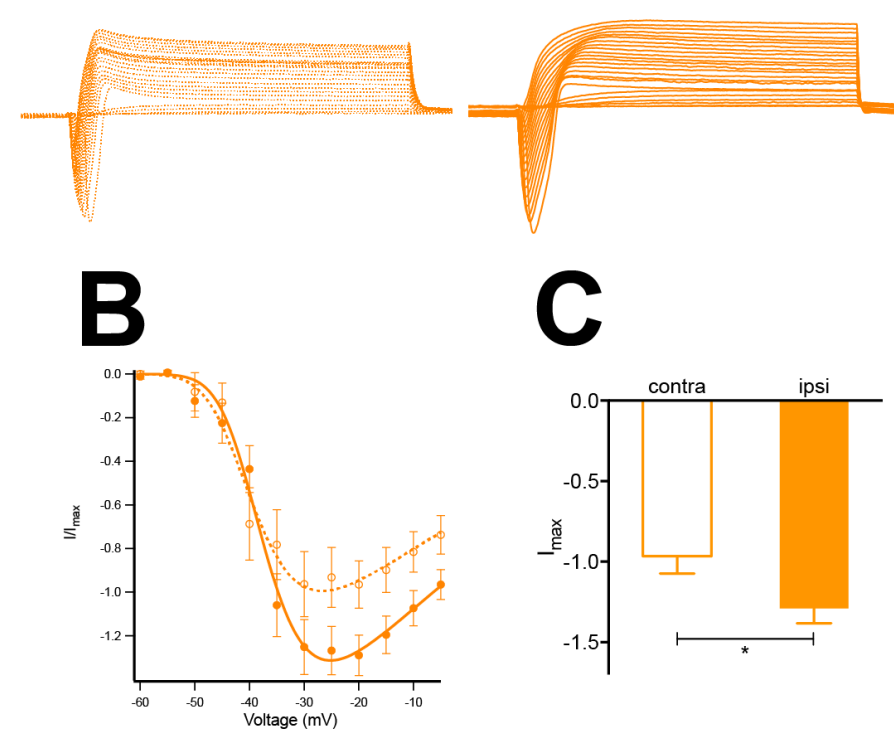



C

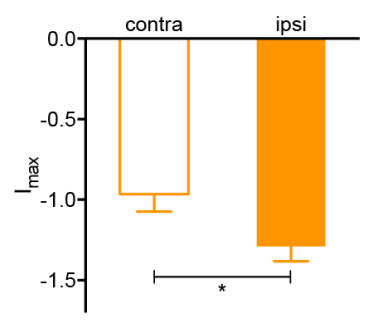

G

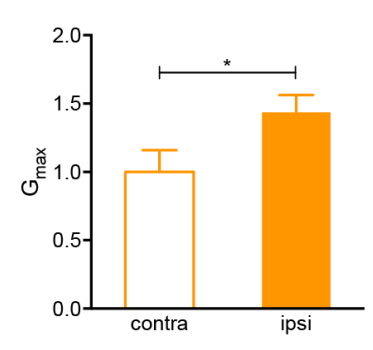

D

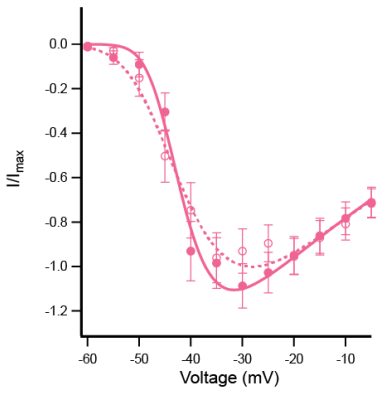

H

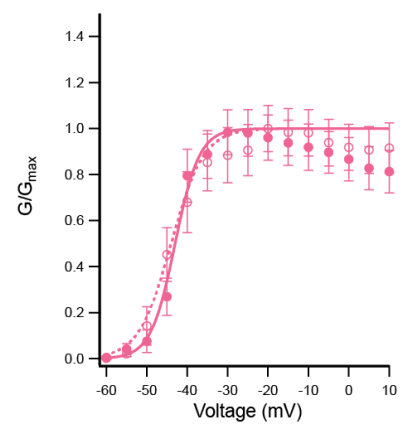

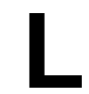



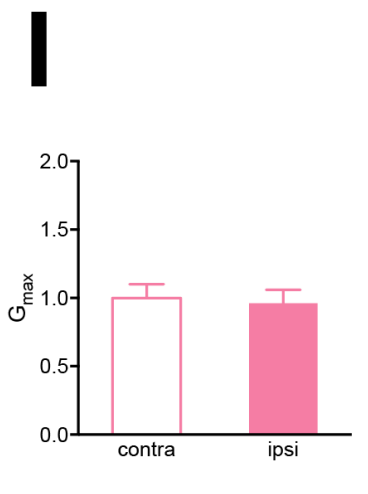

M

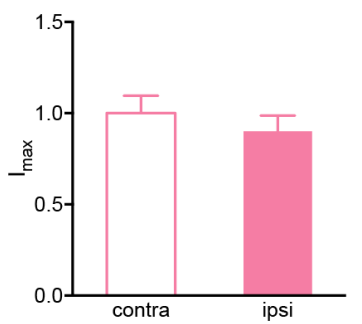

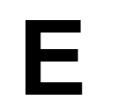

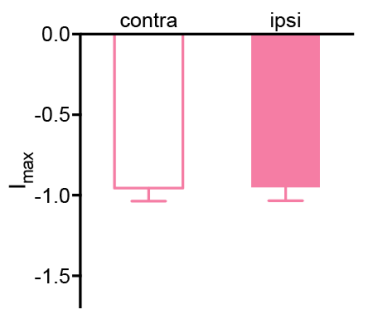


bioRxiv preprint doi: https://doi.org/10.1101/2021.06.16.448689; this version posted June 17 , 2021. The copyright holder for this preprint (which was not certified by peer review) is the author/funder, who has granted bioRxiv a license to display the preprint in perpetuity. It is made available under aCC-BY 4.0 International license.

Figure 6. Changes in sensory neuron macroscopic voltage-gated currents during inflammation. (A) Current traces from IV curve generation for neurons from both ipsilateral and contralateral sides either 4- or 24-hours post-inflammation, representative traces from cells of comparable capacitance. Inward macroscopic current densities normalized to the average peak inward current density of contralateral cells either (B) 4- or (D) 24- hours post inflammation. (C,E) Normalized peak inward current densities. $(\mathbf{F}, \mathbf{H})$ Calculated conductance of inward voltage-gated currents normalized to average peak conductance of contralateral neurons. (G,I) Normalized peak conductance of inward voltage-gated currents. Outward macroscopic current densities normalized to the average peak outward current density of contralateral neurons either (J) 4- or (L) 24- hours post inflammation. (K,M) Normalized peak outward current densities. * $\mathrm{p}<0.05$, unpaired t-test.

comparable at the 24-hour time point (peak normalized current: contra., $-0.96 \pm 0.40$, ipsi., $0.95 \pm 0.44$; peak normalized conductance: contra., $1.00 \pm 0.47$, ipsi., $0.96 \pm 0.50 ;$ Erev: contra., 55.9 $\pm 8.58 \mathrm{mV}$, ipsi., $66.43 \pm 5.18 \mathrm{mV}$; Vhalf: contra., $-43.68 \pm 1.78 \mathrm{mV}$, ipsi., $-45.18 \pm 1.09 \mathrm{mV}$, Figure

\section{Meloxicam relieves hyperalgesia but MoSeq} shows that this pain relief does not equal a return to baseline state.

Having analyzed how primary afferent input is altered, and thus can explain some, but not all of the behavioral changes we observed in the carrageenan inflammatory pain model, we next deployed MoSeq to determine its potential use in the development of effective analgesics. This is because a major current barrier in therapeutic development is the difficulty in assessing analgesia
6A,D-E,H-I). Focusing on outward currents revealed no difference between cells of the contralateral and ipsilateral after 4-hours of inflammation (contra., $1.00 \pm 0.62$, ipsi., $1.20 \pm$ 0.58 , Figure $6 \mathrm{~J}-\mathrm{K}$ ) or 24-hours (contra., $1.00 \pm$ 0.47 , ipsi., $0.90 \pm 0.46$, Figure $6 \mathrm{~L}-\mathrm{M})$.

in an unbiased, rigorous and quantitative manner. First, as a benchmark to current analyses, we began by studying pain relief to carrageenan using the Hargreaves thermal assay. We compared the behavior of mice at 24-hour post-carrageenan injection following intraperitoneal injection of either saline or meloxicam, a non-steroidal antiinflammatory drug (NSAID) commonly used to relieve pain and inflammation in rodents, dogs, cats and humans (Busch et al., 1998) (Figure 7A). As expected, Hargreaves assessment of heat sensitivity shows that meloxicam is efficient at
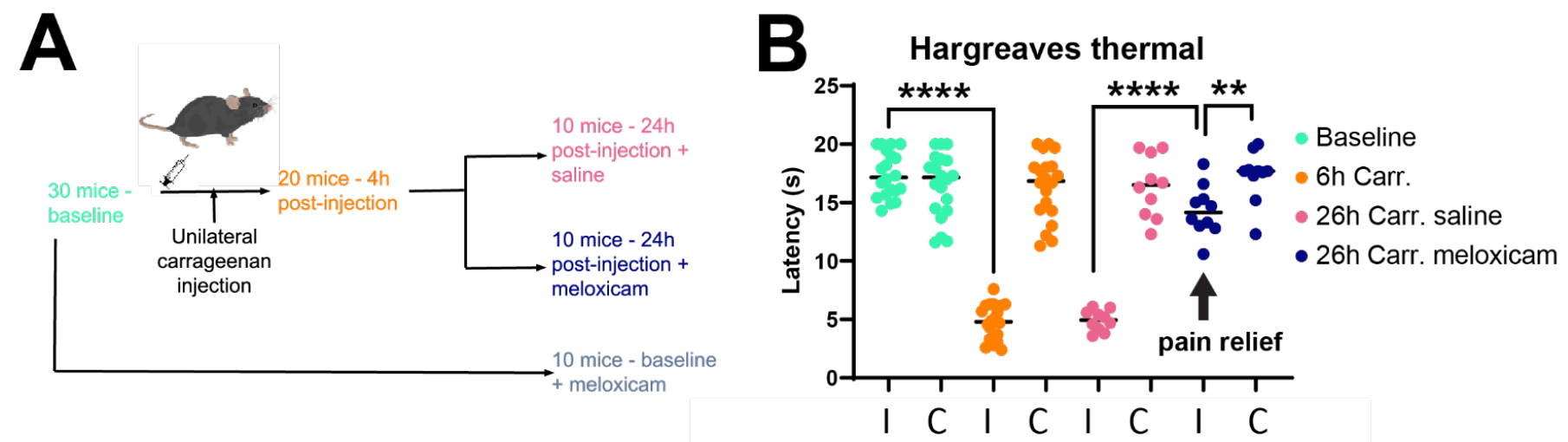

\section{PAWS detects meloxicam pain relief of rapid guarding}
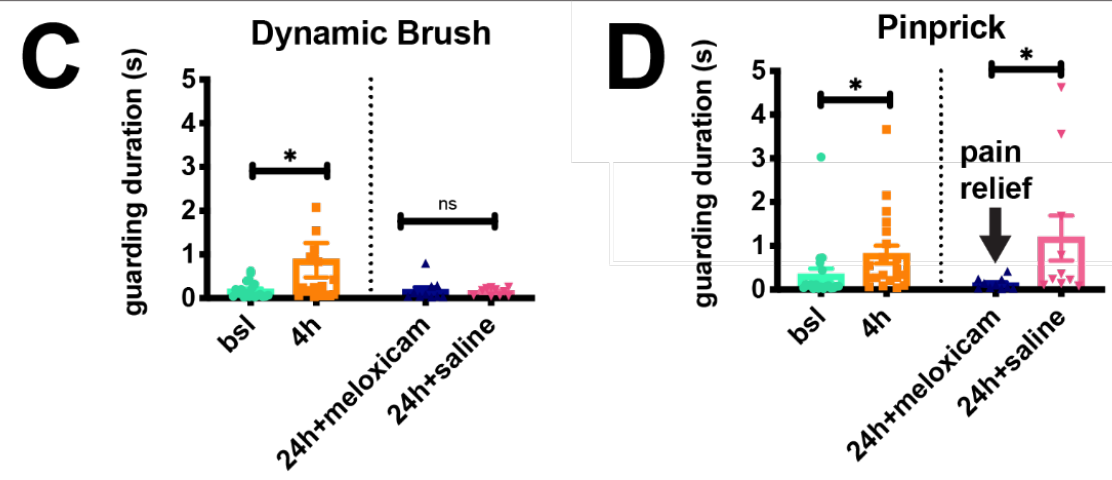
Figure 7. PAWS detects meloxicam pain relief of rapid guarding. (A) Timeline of the experiment. An initial group of 30 mice is tested at baseline with MoSeq and Hargreaves. A subgroup of 20 mice receives an intraplantar injection of $20 \mu \mathrm{l} 3 \%$ carrageenan, is tested at $4 \mathrm{~h}$ post-injection with MoSeq and Hargreaves, then further subdivided into two groups of 10 mice injected intraperitoneally with saline or meloxicam respectively at $22 \mathrm{~h}$ and again tested with MoSeq and Hargreaves at 24h. A control group of 10 mice is injected with meloxicam at baseline and tested with MoSeq and Hargreaves. (B) Hargreaves measurement of carrageenan-induced heat hypersensitivity at baseline, $6 \mathrm{~h}$ following carrageenan injection, as well as pain relief by meloxicam at 26hrs compared to saline. (C,D) Paw guarding duration is measured at baseline, and $4 \mathrm{~h}$ post-carrageenan injection, and $24 \mathrm{~h}$ postcarrageenan injection after saline or meloxicam intraperitoneal injection (striped and solid bar respectively) following dynamic brush (C) or light pinprick (D).

significantly reducing carrageenan-induced heat hypersensitivity at the 24-hour timepoint (Figure 7B, unpaired t-test 24-hour saline vs 24-hour meloxicam $\mathrm{p}<0.0001$ ), though sensitivity is not quite back to baseline levels (unpaired t-test 24hour meloxicam ipsi vs contra $\mathrm{p}<0.01)$.

Before turning to MoSeq, we next returned to highspeed videography combined with the PAWS platform to measure pain relief with meloxicam following carrageenan hind paw injection. We injected carrageenan-inflamed mice with either meloxicam or saline at 22-hours and assessed their mechanical sensitivity using PAWS at baseline, 4hours post-carrageenan injection and 24-hours post-carrageenan intraplantar and compound intraperitoneal injection (Figure 7A). Similar to our observations above, after inflammation, we observed significantly upregulated paw guarding duration evoked by 1) dynamic brush stimulation

We were curious to see whether meloxicam affects the pain signature of spontaneous behavior in a manner similar to evoked sensory behavior, i.e. seemingly bringing animals back to baseline behavior, and used MoSeq to test this hypothesis. Linear discriminant analysis of the usage of the 62 syllables identified by MoSeq shows an interesting directional trend in the behavioral space occupied by mice at baseline, 4- and 24-hours after carrageenan injection, especially when comparing the very different space occupied by mice that have received either saline or meloxicam at 24-hours post-carrageenan injection (Figure 8A, B). Interestingly, animals that received meloxicam at 24-hours do not seem any closer in space to the baseline group than the 24-hour saline control group. That is, conversely to sensory evoked behavior, meloxicam does not appear to show pain reversal in terms of spontaneous behavior (Figure at 4-hours (Figure 7C) (Wilcoxon matched-pairs signed rank test: $4 \mathrm{~h}$ versus baseline, $\mathrm{p}<0.05)$ and 2) light pinprick stimulation at 24-hours postinflammation (Fig 7D) (Wilcoxon matched-pairs signed rank test: $24 \mathrm{~h}$ versus baseline $\mathrm{p}<0.05$ ). Interestingly, when assessing light pinprick evoked behavioral responses of meloxicam- and salinetreated mice, we found that meloxicam administration prevented the progression of carrageenan-induced paw guarding duration that is typically observed at 24-hours after inflammatory pain induction (Wilcoxon matched-pairs signed rank test: 4-hours vs baseline, $\mathrm{p}<0.05$; MannWhitney test, $24 \mathrm{~h}$ carrageenan injection + meloxicam versus $24 \mathrm{~h}$ carrageenan injection + saline, $\mathrm{p}<0.05)$. The circuits that drive paw guarding are the likely result of DRG neuronal activity and some central circuits, and meloxicam might be acting at several points in this node to blunt this behavior.

$7 \mathrm{~F})$. Rather, mice that received meloxicam after injury seem to be in an entirely different "space". This unexpected finding led us to question whether meloxicam can also alter baseline behavior in the absence of pain. While Hargreaves results suggest meloxicam has no effect on the sensory threshold of the uninjected contralateral paw (Figure 7B), MoSeq shows that meloxicam drastically affects spontaneous behavior even at baseline when administered in absence of an underlying pain condition (Figure 7E, F).

We noticed that LDA representations of raw syllable usages (Figure 8A) and transition probabilities (Figure 8B) are slightly different, the pharmacobehavioral groups are more segregated in space when plotting transition probabilities. To better understand and interpret how MoSeq might aid in evaluating analgesic efficacy, we decided to 
bioRxiv preprint doi: https://doi.org/10.1101/2021.06.16.448689; this version posted June 17, 2021. The copyright holder for this preprint (which was not certified by peer review) is the author/funder, who has granted bioRxiv a license to display the preprint in perpetuity. It is made available under aCC-BY 4.0 International license.

\section{MoSeq reveals meloxicam pain relief is not a return to baseline}
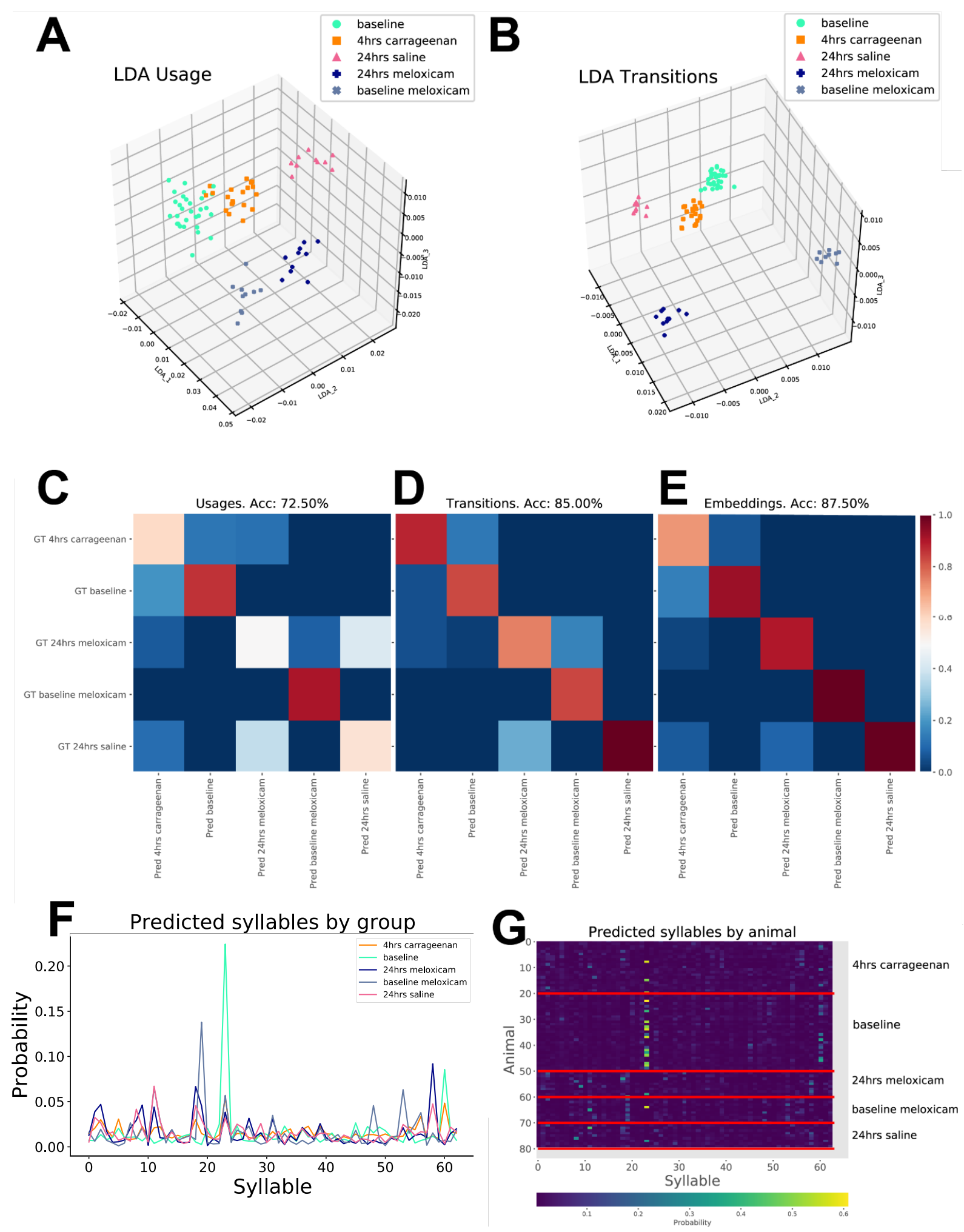

Figure 8. MoSeq shows meloxicam pain relief does not equal return to baseline state. MoSeq data of 5 groups: baseline, baseline + meloxicam intraperitoneal injection, 4h post-carrageenan injection, $24 \mathrm{~h}$ post-carrageenan injection + saline intraperitoneal injection, 24h post-carrageenan injection + meloxicam intraperitoneal injection. (A, B) Representation of linear discriminant analysis (LDA) of raw usage $(\mathbf{A})$ or transition probability $(\mathbf{B})(\mathbf{C}, \mathbf{D}, \mathbf{E})$ Held-out data confusion matrices for an identical classifier trained on raw usage data (C), transition probabilities (D) or learned embedding (E) with leave-one-out cross validation, Acc.=accuracy. $(\mathbf{F}, \mathbf{G})$ Representation of the syllables predicted to be most representative of groups (F) or animals (G), based on learning an embedding space for co-occurring syllables and animals. For example syllable 54 is most representative of the baseline state both in $\mathrm{F}$ and $\mathrm{G}$.

apply standard sequence classification techniques (Le and Mikolov, 2014) to MoSeq data to extract syllables or sets of syllables which best represent a particular experimental group (baseline, 24-hour 
saline or 24-hour meloxicam pain relief, for example). These methods embed long sequences (e.g., the raw MoSeq data produced by one animal) in a representational space where sequences having similar co-occurrence structure (pairs of syllables, triples of syllables, etc.) tend to cluster. Consequently, these embeddings depend on contextual information which is potentially more powerful than simple transition probabilities and is simply absent in usages. To evaluate the relative predictive powers of usages versus transition probability versus learned embeddings, we trained three classifiers to predict experimental groups from each type of representation. We found that the context-dependent, learned embeddings were substantially more predictive of experimental groups than raw usages or transition probabilities (72.5\% accuracy for raw usages; $85 \%$ accuracy for transition probabilities; $87.5 \%$ accuracy for learned embeddings; full confusion matrices depicted in Fig. 8C, C, E).

Crucially, the learned embedding method associates to each point in the embedding space a distribution over syllables. This is gradually shaped through training to indicate behaviors associated to each embedded sequence (i.e., to each animal) and plotting these distributions per animal after

\section{Discussion}

Unilateral intraplantar injection of carrageenan evokes both mechanical and thermal hyperalgesia which persist for at least 24-hours (Hargreaves et al. 1988; Buisseret et al. 2019). Here, with videography across timescales followed by unbiased analyses using machine learning based algorithms, we identify evoked and spontaneous behaviors after induction of inflammation that were previously undetected with traditional methods for assessing nociception. Profiling sensory neurons directly innervating the site of inflammation revealed hyperexcitability after 4-hours of inflammation, which resolved to a basal level at the 24-hour time point. Moreover, expression of the heat-gated ion channel TRPV1 was elevated at 24-hours suggesting a longer-lasting adaptation of nociceptors following the inflammatory insult. training gives Figures 8F, G. While LDA representation of raw syllable usage or transition probabilities can give us a global idea of how mouse spontaneous behavior is affected by pain and pain relief across time, learned embeddings now provide us with the means to identify syllables that are most representative of each pharmacobehavioral state. For example, we can now identify syllables 23 and 60, a type of exploratory behavior and grooming respectively, as possibly representative of spontaneous behavior at baseline, but less so at 4hours post-carrageenan injection when the animal is in pain, and not at all at 24-hours. We also see that syllable 19, a head-up micro-movement, is representative of mice having received meloxicam either at baseline or at 24-hours post-carrageenan injection. When using this information to look at syllable usage, we find that this behavior is indeed significantly down-regulated in both groups compared to baseline, potentially highlighting a side-effect of meloxicam. In conclusion, we have identified the learned embeddings method as a crucial tool in order to extract biologically meaningful data from rich and complex MoSeq datasets. This type of analysis will act as a guide for the experimenter to identify specific behaviors representative of pain and pain relief states.

Noteworthy to the quest for discovering new analgesics, we determined that meloxicam driven pain relief does not equate to a full return to preinflammatory baseline state. Taken together, these data highlight the strength of this multidisciplinary approach for arraying the highdimensional pain biobehavioral space and offer a new experimental roadmap for the field.

\section{Resolving the transition between responses at 4- versus 24-hours}

A single injection of carrageenan produces localized inflammation which resolves within a day, but pain-like behavior that can persist for days. However, our multi-method pipeline shows that DRG neurons innervating the site of inflammation 
do not show signs of hyperexcitability 24-hours after inflammation. This suggests the persistent pain behaviours resolved with MoSeq, PAWS, and B-SOiD at the 24-hour time point are likely driven by adaptations in the nociceptive system initiated during active inflammation. Indeed we show an increase in the number of sensory neurons expressing TRPV1 24-hours post-inflammation and thus postulate that the sensory neurons exist in a hyperexcitable state, even as the inflammation subsides. Hence the observation of pain-like behaviours at both our early and later time points, which we have resolved through MoSeq and PAWS as distinct biobehavioral states. Another study identified a key role for the TRPA1 channel in mediating carrageenan-induced paw edema (Moilanen et al., 2012), suggesting that sensitization of several nociceptive ion channels likely contribute to the pain hypersensitivity state. It is also plausible that central sensitization contributes to the persistent pain observed 24hours after inflammation, although this could only be assessed through simultaneous recording of neurons in the brain and/or spinal cord.

\section{Retrograde labelling allows interrogation of the cellular mechanisms driving biobehavioral states}

Using the retrograde tracer Fast Blue we demonstrate higher excitability of sensory neurons innervating the inflamed hind paw after 4-hours of inflammation. Subsequent experiments exploring the activity of voltage-gated ion channels revealed a greater conductance of those channels permitting inward current in response to depolarisation, which may offer some explanation of the hyperexcitable state observed, as action potential discharge can be more readily achieved. The rapid onset of this change suggests a post-translational modification might be responsible, indeed phosphorylation of voltage-gated sodium channels by mediators released during inflammation, including prostaglandin E2 (PGE2), has been shown to increase inward currents (Gold et al., 1996). Considering that meloxicam inhibits cyclooxygenase activity and thus prostaglandin production, our finding that meloxicam reduced pain like behavior 4-hours post injection of carrageenan suggests a strong role of PGE2induced phosphorylation of voltage-gated sodium channels driving the hyperexcitability of sensory neurons and pain behaviours that manifest following induction of the carrageenan model. Furthering this, independent studies have reported induction of cyclooxygenase-2 during the hind paw carrageenan model and less pain-like behavior following induction of the model on mice deficient in the voltage-gated sodium channel $\mathrm{Na}_{\mathrm{V}} 1.7$ on nociceptors (Ibuki et al., 2003; Nassar et al., 2004). Using Fast Blue to identify the cell bodies of sensory neurons projecting to the inflamed hind paw we report an apparent upregulation of TRPV1 by lumbar DRG through immunohistochemistry after 24-hours of inflammation. Studies of TRPV1 knock-out mice have also reported less mechanical hyperalgesia following carrageenan-induced inflammation (Watanabe et al., 2015), we thus propose the observed increase contributes to painlike behaviours seen at 24-hours. Combining retrograde labelling with more sophisticated techniques such as single-cell qPCR or RNA sequencing would allow a more comprehensive assessment of the molecular state of sensory neurons during inflammation, removing the requirement of validated antibodies and in the latter case may represent an opportunity to identify novel players in inflammatory pain networks at the level of the sensory neuron.

\section{Supervised and unsupervised learning approaches uncover behavioral signatures that can distinguish mechanical hyperalgesia and allodynia.}

Using the PAWS platform, we determined that after carrageenan-induced hind paw inflammation, rapid reflexive behaviors related to how fast and high the withdrawn paw is moved, are unaltered. This finding likely reflects the fact that animals experiencing localized paw inflammation do not want to move their swollen paws robustly, which would facilitate ensuing pain. Conversely, with PAWS, we observe an upregulation of defensive coping behaviors like paw guarding to both innocuous and noxious mechanical stimulation. This result suggests that animals experiencing inflammatory pain are willing to engage in motor 
behaviors that have the potential to prevent further stimulation to the injured paw (i.e. guarding the paw in the air). The rapid reflexes are likely predominantly DRG and spinally mediated, whereas defensive coping behaviors have central components (Barik et al., 2018; Corder et al., 2019; Hua et al., 2020; Huang et al., 2019; Jones et al., 2020) in structures like the amygdala and brainstem. Moreover, recent studies uncovered an ascending spinoparabrachial pathway where Tacr1 or Gpr83 projection neurons can encode the negative valence associated with noxious stimuli (Choi et al., 2020). Based on our data presented here, we speculate that the carrageenan model might specifically sensitive molecularly-defined pathways such as these, and future studies are warranted to test this hypothesis.

Taken together, our results demonstrate that PAWS can accurately make a distinction between strictly reflexive and affective centrally-modulated behavioral responses to mechanical stimulation under pathophysiological conditions, both in a time-specific manner and with respect to sensory modalities. In addition, the B-SOiD unsupervised learning algorithm was able to identify variations of the guarding response that were specific to delivery of innocuous versus noxious stimuli. Future studies are needed to determine how animals engage unique paw motor patterns during inflammatory pain, but this must reflect something about the underlying neural circuits. To our knowledge, this is the first demonstration that the paw guarding coping mechanism is specific to given mechanosensory modalities and nuanced yet robust paw position dynamics allow the fine distinction between dynamic mechanical allodynia and mechanical hyperalgesia.

\section{Unsupervised 3D pose dynamics show spontaneous pain signatures}

Gait analysis shows that weight bearing of the injected paw is significantly affected at the 4-hour time point after carrageenan injection but not at the 24-hour time point, suggesting that either the pain has started to recede, which is contradicted by evoked pain measures, or the animal has adapted somewhat to the pain state. Interestingly,
MoSeq analysis of the animal's spontaneous behavior also shows two distinct trends in how the establishment of pain affects usage of specific syllables. While animals use certain micromovements less and less (or more and more) as hyperalgesia progresses, other syllables usage shows rather a U-shaped curve. The fact that the usage of certain movements is most affected during the acute phase and tends to revert back to preinjury levels at 24-hours suggests adaptation of motor behavior to pain. Comparing rearing behavior in a cylinder over a few minutes to the deeper analysis provided by MoSeq outlines well this dichotomy. While overall time spent rearing at 24-hours post-carrageenan injection looks similar to baseline, freely moving mice filmed for 20 minutes will actually show different types of rearing behavior, some of which are only affected at 4 -hours by pain, while others are significantly down-regulated at both 4- and 24-hours.

Our analysis of predicted syllables per group shows that each pharmacobehavioral state (baseline state, different pain states, pain relief...) has a distinct signature. Interestingly, while meloxicam relieves hyperalgesia entirely when we use evoked sensory tests to assess pain relief, we see that its effect on spontaneous behavior is more subtle and that mice injected with either saline or meloxicam at 24-hours post-carrageenan injection still share common predicted syllables. Hence, similarly to RNA sequencing of neurons, where a neuronal subset is not defined by one specific gene but rather by a set of genes of varying levels of expression, the signature of a pharmacobehavioral space is really a set of behavioral syllables characterized by their levels of usage.

Our comparisons of the predictive power of raw syllable usage, transition probabilities between syllables or learned representations highlights an interesting point about how these different parameters can be used to extract the most representative set of data. When tested on a leaveone-out cross-validation, we find that using learned embeddings gives a significantly more descriptive representation of our datasets than syllable usage or transition probabilities. But these approaches are actually complementary because while LDA representation of usages or transitions gives us a 
broad picture of how behavior is impacted by pain and pain relief, learned embeddings allow us to identify in a meaningful and biologically relevant way the micro-movements most representative of pain and analgesic states. We can then use how these micro-movements as a tangible readout of the analgesic potential of new drugs.

\section{What is analgesia?}

Analgesia is broadly defined as a lack of pain or an insensitivity to pain, and the International Association for the Study of Pain describes both a sensory and an emotional component to the experience of pain. Hence, analgesia could be defined as a relief of both sensory-evoked and ongoing emotional pain, but is that the same as a return to pre-injury state or a different state altogether?

Mice are hypersensitive to heat and pinprick at 4and 24-hours post-carrageenan injection and this hyperalgesia is entirely relieved by meloxicam, which may seem like a return to a pre-injury state. However, Motion Sequencing shows that this evoked pain relief does not equal a return to baseline spontaneous behavior, rather this is an entirely different state and though meloxicam relieves hypersensitivity to noxious stimulation, it does not bring the animal back to a pre-injury behavioral state, nor probably a pre-injury internal state. While MoSeq analysis of mouse spontaneous behavior uncovers aspects of pain and pain relief that cannot be identified through evoked sensory testing alone, the internal state of the animal is still a mystery and it would be interesting to investigate further.

One of the most surprising findings of our study is the drastic effect at baseline of an antiinflammatory drug widely used on animals, as shown by Motion Sequencing of their spontaneous behavior. Prostaglandins are well known mediators of acute inflammation, and recent studies also suggest a role in the transition from acute to chronic inflammation (Aoki and Narumiya, 2012). Meloxicam is a non-steroidal anti-inflammatory drug widely used in research laboratories using rodent models, as well as in the clinic, to reduce pain and fever symptoms of inflammatory pain by reducing inflammation-induced prostaglandin synthesis. Less inflammation-induced prostaglandin synthesis translates into lesser sensitization of the nerve fibers innervating the injury site, as well as the central nervous system. This is in line with the mechanical and thermal hypersensitivity relief we see using evoked pain tests, seemingly bringing animal sensitivity almost back to baseline levels. Our analysis of spontaneous behavior and representation of the results in $3 \mathrm{D}$ space however tells a different story. Following meloxicam injection, although mice show evoked pain relief, they are in a very different pharmacobehavioral state compared to baseline. This suggests that while sensory-evoked pain might be relieved, there is an ongoing affectivemotivational component at play that is altered in an unexpected way by meloxicam. For this reason, and because many people could assume a lack of effect of an anti-inflammatory drug in the absence of inflammation, we decided to evaluate whether meloxicam affects spontaneous behavior pre-injury through MoSeq. We found that spontaneous mouse behavior is significantly affected by meloxicam at baseline, and in many ways, this effect is even more drastic at baseline than in a pain context. While in a pain state meloxicam mainly inhibits the inflammation-induced activity of the prostaglandin-endoperoxide synthase COX-2, the constitutive activity of the COX-1 isoform is also affected. We suggest that this is one of the mechanisms underlying the effect of meloxicam at baseline. Considering the power of MoSeq in representing evolving pain states, it will be interesting to use this technology to follow the timeline of other more complex rodent pain models, but also to test the analgesic potential of drug candidates and redefine what we call analgesia.

\section{Materials and Methods}

\section{Animals (Rutgers University)}

Male mice of C57BL/6J background were used for behavioral analyses. Testing was done beginning at 7 weeks of age and completed by 12 weeks of age. All animals were group housed. Animal numbers per group for behavioral tests are indicated in figures. All procedures were approved by the 
Rutgers University Institutional Animal Care and Use Committee (IACUC; protocol \#: 201702589). All mice used in experiments were housed in a regular light cycle room (lights on from 08:00 to 20:00) with food and water available ad libitum. 20 pl $3 \%(\mathrm{w} / \mathrm{v}) \quad \lambda$-Carrageenan (Sigma-Aldrich) in PBS 1X was injected into the mouse left hind paw using a Hamilton syringe. Thermal hyperalgesia was assessed at baseline and 6- and 26-hours postcarrageenan injection. The uninjected right hind paw served as a control. Saline or meloxicam (5mg/kg, Henry Schein Animal Health) was injected intraperitoneally 22 hours postcarrageenan injection (2 hours before MoSeq, 4 hours before Hargreaves).

\section{Animals (University of Cambridge)}

Experiments performed in Cambridge UK (dynamic weight bearing, electrophysiology and immunohistochemistry) were regulated under the Animals (Scientific Procedures) Act 1986 Amendment Regulations 2012. The University of Cambridge Animal Welfare and Ethical Review Body also approved all animal experiments. Cutaneous afferents innervating both hind paws of male C57BL/6J mice were labelled with the retrograde tracer Fast Blue $(2 \% \mathrm{w} / \mathrm{v}$ in sterile PBS; Polysciences) when animals were aged 9 weeks. $3 \times 1 \mathrm{\mu l}$ injections were made to the lateral, central and medial plantar aspects of each hind paw under anaesthesia (intraperitoneal delivery of ketamine, $100 \mathrm{mg} / \mathrm{kg}$ and xylazine, $10 \mathrm{mg} / \mathrm{kg}$ ) as previously described (da Silva Serra et al., 2016). 5-7 days after retrograde labelling, the right hind paw of mice was inflamed by injection of $20 \mathrm{\mu l} 3 \%$ $(\mathrm{w} / \mathrm{v}) \quad \lambda$-carrageenan (Sigma-Aldrich), inflammation was induced while mice were under inhalation anaesthesia ( $2 \%$ isoflurane). The diameters of each ankle and foot pad were measured with digital callipers before and 4- or 24hours post inflammation. 5 animals were used for each time point for electrophysiology experiments, a separate cohort of 4 animals were used for immunohistochemistry experiments.

\section{Animals (University of Pennsylvania)}

Mice for behavior testing were maintained in a barrier animal facility in the Carolyn Lynch building at the University of Pennsylvania. The Lynch vivarium is temperature controlled and maintained under a 12-hr light/dark cycle ( $7 \mathrm{am} / 7$ pm) at 70 degrees Fahrenheit with ad lib access to food (Purina LabDiet 5001) and tap water. The feed compartment on the wire box lid of the cage was kept at a minimum of $1 / 3$ full at all times. All cages were provided with nestlets to provide enrichment. All procedures were conducted according to animal protocols approved by the university Institutional Animal Care and Use Committee (IACUC) and in accordance with the National Institutes of Health (NIH) guidelines. C57BL mice were purchased from Jackson Laboratories. All animals were habituated to our facility for 2 weeks after delivery before beginning behavioral experiments described below. All mice were adults between 2 and 4 months. Animals were co-housed with $4-5$ mice per cage in a large holding room containing approximately 500 cages of mice. $20 \mathrm{ll} 3 \%$ (w/v) $\lambda$-Carrageenan (Sigma-Aldrich) in $0.9 \%$ sterile $\mathrm{NaCl}$ solution (saline) was injected into the mouse hind paw. Mechanical sensitivity was assessed with PAWS at baseline, 4 and 24 hours post carrageenan injection. Inflammation was induced while mice were under inhalation anaesthesia (2 to $3.5 \%$ isoflurane according to mice's loss of consciousness and anaesthetic depth (monitoring of respiratory rate and pattern and responsiveness to toe pinch). Saline or meloxicam (5mg/kg, Henry Schein Animal Health) was injected intraperitoneally 22 hours postcarrageenan injection (2 hours before the last PAWS behavioral testing session).

\section{Motion Sequencing (MoSeq): Motion Sequencing}

Depth data were modelled as previously published (Wiltschko et al., 2015). First, raw depth frames were collected from a Microsoft Kinect, mounted above the arena, using custom acquisition software written in $\mathrm{C \#}$. Frames were collected at $30 \mathrm{~Hz}$, and each frame was composed of 512 x 424 pixels, where each pixel contained a 16-bit integer 
specifying the distance of that pixel from the sensor in $\mathrm{mm}$. After each session, frames were gzip compressed and moved to another computer for offline analysis. The mouse's center and orientation were found using an ellipse fit. Then, an 80 x 80 pixel box was drawn around the mouse, and the mouse was rotated to face the right hand side. Next, if the tracking model was used, missing pixels were identified by their likelihood according to the Gaussian model. Low-likelihood pixels were treated as missing data and principal components (PCs) are computed using probabilistic PCA (Roweis, 1998; Tipping and Bishop, 1999). Finally, frames were projected onto the first $10 \mathrm{PCs}$, forming a 10 dimensional time series that described the mouse's $3 \mathrm{D}$ pose trajectory. These data were used to train an autoregressive hidden Markov model (ARHMM) with 3 lags to cluster mouse behavioral dynamics into discrete "syllables," with state number automatically identified through the use of a hierarchical Dirichlet process. Each state was comprised of a vector autoregressive process that captures the evolution of the 10 PCs over time. The model was fit using Gibbs sampling as described in (Wiltschko et al., 2015) using freely available software (https://github.com/mattjj/ pybasicbayes). Model output was insensitive to all but two hyperparameters, which were set using unsupervised techniques for determining the length scales for discrete behaviors as was previously published (Wiltschko et al., 2015).

\section{Motion Sequencing (MoSeq): Behavioural usage and transition matrix analysis}

Syllable usage was calculated by summing the number of occurrences of each syllable and dividing by total syllable usage across a recording session, converting syllable usage into a percentage. The number of syllables used for each analysis was based on the syllable usage across all sessions within a condition. Transition matrices were calculated by counting the total number of occurrences syllable A transitions into syllable B (for all syllables).

\section{Motion Sequencing (MoSeq): Behavioral Linear Discriminant Analysis}

Linear Discriminant Analysis (LDA) was performed using the scikit-learn implementation.
Individual normalized usage or bigram transition probabilities were fed to the LDA model, including group labels, and fit to either 2 or 3 components using the eigen solver and an empirically found shrinkage value. Results were then plotted with seaborn and matplotlib.

\section{Learned Embeddings of Syllable Sequences}

Each animal was represented as a doc2vec ("document to vector") embedding [1] using the Gensim software package [2], version 3.8.3, in Python 3. To that end, each animal and each syllable was first encoded uniquely as a one-hot vector of 164 (80 animals +64 syllables; four syllables of the 68 total were removed for occurring fewer than 10 times on average in the raw sequences) dimensions. These one hot vectors were mapped by a linear transformation to an $n$ dimensional embedding space. The linear transformation was adapted by stochastic gradient descent using two losses, resulting in two embeddings. Following [1], the two embeddings for each animal were concatenated to produce the final animal representation. The first embedding was learned by 1) randomly sampling an animal and a single syllable from its raw behavioral sequence, 2) mapping them to an $n=100$ dimensional embedding space, 3) averaging the animal and syllable embeddings, 4) mapping the average to the 64-dimensional space of probability distributions over syllables, and 4) predicting from this output distribution either the previous syllable or next syllable in the raw sequence. The embedding space was adapted to improve accuracy in this syllable prediction task. In the parlance of doc $2 \mathrm{vec}$ methods, this corresponds to a "distributed memory" (DM) embedding with a window size of 1. The second embedding was learned using only representations of animals (no syllable embeddings), by first randomly sampling an animal together with a 10-syllable subsequence in its raw behavioral sequence and mapping the animal to an $n=50$ dimensional embedding space. The animal embedding was then mapped to the 10x64 dimensional space of distributions on 10syllable subsequences, from which random syllables in the sampled subsequence window were predicted. The embedding space was adapted to improve the accuracy of this subsequence 
prediction task, which also helps the embedding encode higher order information beyond neighboring syllables in the raw sequence. We refer to this as a "distributed bag of words" (DBOW) embedding. Again, the final embedding for each animal to be used in classification was the concatenation of DM and DBOW embeddings.

We determined by inspection that the default doc2vec hyperparameters ultimately produced the best classifier performance. The model was trained over 150 epochs, and each epoch consisted of one gradient descent update for every 2-syllable window for every animal in the DM representation and every 10-syllable window for every animal in the DBOW representation. Both the number of training epochs and the embedding dimension are nonstandard for doc2vec applications, but they nevertheless produced the best classifier results. 150 epochs is longer than in other applications since we are only concerned with overfitting at the classifier stage (e.g. there is no testing data for the learned embeddings). There is also the danger of overfitting from using more embedding dimensions than total syllables (100 vs 64 ) in the DM model. We circumvent this problem since the DM model has 80 additional data in the form of animals so that the eventual joint DM/DBOW embedding is less susceptible to overfitting.

There are several ways to associate to each embedding a characteristic syllable or sequence of syllables. One way, not explored here, is to use the syllable embeddings produced by the DM representation and calculate some similarity metric (cosine similarity, Euclidean distance, etc.) between a syllable embedding and an animal embedding. Instead, to provide a richer association between animals and characteristic behaviors, we modified the Gensim source code to expose the mapping from the embedding space to the 64dimensional syllable space used during training in the DM model. This mapping can now be interpreted as an association between an animal representation and a distribution of syllables. This association was fairly stable over hyperparameters and high classifier performance was associated with strongly peaked associated syllable distributions. The presented method is a proof of a concept for the viability of sequence embedding methods for the explanation of animal behavior, and future work could cross-reference "explanatory" syllables against ethological prior knowledge and use these syllables to make concrete behavioral hypotheses.

\section{Classifier Analysis of Animal Representations}

We used a machine learning classifier to compare the expressive power of three types of animal representations: syllable usages, syllable transition probabilities, and learned embeddings. Our goal here was to predict which of the five experimental manipulations an animal received from each of these representations. By design, the dimensionality of each representation is different ( 68 vs 150 vs $68 \times 68=4624$ ), but can be truncated to make a fair comparison. In general, the higher the dimension of the data, the easier it will be to classify since more dimensions give more degrees of freedom for a learned decision boundary. To start, we fixed the representational dimensions at their normal values, giving transition matrices a massive advantage. We trained an identical, unregularized, 5-class logistic regression classifier on each type of representation using leave-one-out crossvalidation. The classifier was trained in scikit-learn in Python 3. The classifier was trained with a stopping criterion of $1 \mathrm{e}-5$ and with balanced class correction. In this setting, the average validation accuracy for the usages, transitions and embeddings were $72.5 \% 85 \%$ and $87.5 \%$, respectively. If we fix the dimensions of representations to all be 68 (taking the 68 most frequent transitions and setting $n=34$ for the embeddings), the accuracies associated to transitions and embeddings drop by $22.5 \%$ (to $62.5 \%$ ) and $10 \%$ (to $77.5 \%$ ), respectively. This shows that the learned embeddings not only perform the best with the standard representational dimensions, but are also the most efficient of the three tested representations. We can hypothesize consequently that there are experimental effects which differentiate the groups which manifest at longer behavioral scales (beyond one-step transitions).

\section{Behavior: Hargreaves Assay}


To assess hind paw heat sensitivity, Hargreaves' test was conducted using a plantar test device (IITC). Mice were placed individually into Plexiglas chambers on an elevated glass platform and allowed to acclimate for at least 30 minutes before testing. A mobile radiant heat source of constant intensity was then applied to the glabrous surface of the paw through the glass plate and the latency to paw withdrawal measured. Paw withdrawal latency is reported as the mean of three measurements for both hind paws with at least a 5 min pause between measurements. A cut-off of 20 $\mathrm{s}$ was applied to avoid tissue damage.

\section{Behavior: Dynamic Weight Bearing}

The weight bearing of free-moving animals was assessed using a dynamic weight bearing apparatus (Bioseb). Each test lasted three minutes, mice were naïve to the test device before baseline weightbearing was assessed. The 2 highest confidence levels of automatic paw assignment by the accompanying software were taken forward for analyses; correct paw assignment was manually validated for at least 1 minute 30 seconds of each test.

\section{Electrophysiology}

Mice were sacrificed by cervical dislocation before the lumbar DRG (L2 - L5) from carrageenaninjected and non-injected sides were collected separately in dissociation media (L-15 + GlutaMAX growth media supplemented with 24 $\mathrm{mM} \mathrm{NaHCO}$; Life Technologies). Dissected DRG were then incubated in dissociation media containing $1 \mathrm{mg} / \mathrm{ml}$ type $1 \mathrm{~A}$ collagenase (SigmaAldrich) and $6 \mathrm{mg} / \mathrm{ml}$ bovine serum albumin (BSA; Sigma-Aldrich) for 15 min at $37^{\circ} \mathrm{C}, 5 \% \mathrm{CO}_{2}$, before a further $30 \mathrm{~min}$ in dissociation media containing $1 \mathrm{mg} / \mathrm{ml}$ trypsin (Sigma-Aldrich) and 6 $\mathrm{mg} / \mathrm{ml} \mathrm{BSA}$. DRG were then suspended in culture media (L-15 + GlutaMAX growth media supplemented with $10 \%(\mathrm{v} / \mathrm{v})$ foetal bovine serum, $24 \mathrm{mM} \mathrm{NaHCO} 38 \mathrm{mM}$ glucose and $2 \%(\mathrm{v} / \mathrm{v})$ penicillin/streptomycin) before several rounds of mechanical trituration and brief centrifugation (160g, $30 \mathrm{~s}$ ). After sufficient trituration, dissociated cells were pelleted (160g, $5 \mathrm{~min}$ ), resuspended in culture media and plated on poly-D-lysine/laminin coated glass coverslips (BD Biosciences) and incubated at $37{ }^{\circ} \mathrm{C}, 5 \% \mathrm{CO}_{2}$. Electrophysiology experiments were performed the following day, recordings were made using an EPC-10 amplifier (HEKA) and corresponding Patchmaster software. The extracellular solution contained (in $\mathrm{mM}$ ): $\mathrm{NaCl}(140), \mathrm{KCl}(4), \mathrm{MgCl}_{2}(1), \mathrm{CaCl}_{2}$ (2), glucose (4) and HEPES (10), pH 7.40. Patch pipettes were pulled from borosilicate glass capillaries (Hilgenberg) using a P-97 pipette puller (Sutter Instruments) with resistances of 4-8 $\mathrm{M} \Omega$ and backfilled with intracellular solution containing (in $\mathrm{mM}$ ): $\mathrm{KCl}$ (110), $\mathrm{NaCl}(10), \mathrm{MgCl}_{2}$ (1), EGTA (1), HEPES (10), $\mathrm{Na}_{2} \operatorname{ATP}(2), \mathrm{Na}_{2} \mathrm{GTP}$ (0.5), pH 7.30. Whole cell currents or voltages were sampled at 20 $\mathrm{kHz}$ from Fast Blue labelled neurons, identified by LED excitation at $365 \mathrm{~nm}$ (Cairn Research). Stepwise depolarisation $(\Delta 10 \mathrm{pA}, 50 \mathrm{~ms})$ was used to determine the action potential threshold of cells. Only cells which fired action potentials and had a resting membrane potential less than or equal to $40 \mathrm{mV}$ and were included in analyses. Action potential parameters were measured using Fitmaster software (HEKA) and IgorPro software (Wavemetrics) as previously described (\{Chakrabarti, 2018 \#629\}. The excitability of neurons was further assessed by applying a suprathreshold (2x action potential threshold) for $500 \mathrm{~ms}$, the frequency of action potentials during this time was noted. The activity of macroscopic voltage-sensitive channels was assessed in voltageclamp mode with appropriate compensation for series resistance. Cells were held at $-120 \mathrm{mV}$ for $150 \mathrm{~ms}$ before stepping to the test potential (-60 $\mathrm{mV}-55 \mathrm{mV}$ in $5 \mathrm{mV}$ increments) for $40 \mathrm{~ms}$ and returning to a holding potential of $-60 \mathrm{mV}$ for 200 ms between steps. Peak inward and outward currents were normalised to cell size by dividing by cell capacitance. Peak inward current densities were then fit to a Boltzmann function to determine the reversal potential and half-activating potential of voltage sensitive channels. The conductance of channels guarding inward currents was determined using the equation, $\mathrm{G}=\mathrm{I} /\left(\mathrm{V}_{\mathrm{m}}-\mathrm{E}_{\text {rev }}\right)$, where $\mathrm{G}$, conductance; I, peak inward current; $\mathrm{V}_{\mathrm{m}}, \mathrm{mV}$ step to elicit $I$ and $E_{\text {rev }}$, reversal potential and fit to a Boltzmann function. To compare macroscopic voltage-sensitive currents between neurons isolated from the inflamed and non-inflamed sides peak current densities and conductance values were 
normalised to those obtained from cells from the contralateral side.

\section{Immunohistochemistry}

24-hours post inflammation a cohort of 4 mice were transcardially perfused with $4 \%$ (w/v) PFA under terminal anaesthesia (intraperitoneal delivery of $200 \mathrm{mg} / \mathrm{kg}$ sodium pentobarbital). Lumbar DRG (L3 - L4) were then collected from both the inflamed and non-injected sides and post-fixed in Zamboni's fixative for $30 \mathrm{~min}$, followed by overnight incubation in $30 \%(\mathrm{w} / \mathrm{v})$ sucrose at $4{ }^{\circ} \mathrm{C}$ for cryoprotection. Individual DRG were then snap-frozen in Shandon M-1 Embedding Matrix (Thermo Fisher Scientific). $12 \mu \mathrm{m}$ sections of each DRG were collected on a cryostat sequentially across 10 slides. After washing with PBS containing $0.001 \%$ (v/v) Tween-20 (Thermo Fisher Scientific), slides were incubated with antibody dilutant $(1 \%(\mathrm{w} / \mathrm{v})$ BSA, $5 \%(\mathrm{v} / \mathrm{v})$ donkey serum and $0.02 \%(\mathrm{v} / \mathrm{v})$ Triton-X-100 in PBS) at room temperature for 1 hour before overnight incubation at $4{ }^{\circ} \mathrm{C}$ with an anti-TRPV1 antibody (1:500; guinea-pig polyclonal; Alomone, AGP-118). The following day slides were washed three times with PBS-Tween before incubation with donkey antiguinea-pig IgG-AF488 for 2 hours at room temperature, followed by a further two washes and mounting. Images were acquired with an Olympus BX51 microscope and Q-Imaging camera. Two sections per DRG per animal were analysed, briefly, each cell was selected as a region of interest and individual cells were considered positively stained if the background corrected intensity exceeded $2 \mathrm{x}$ SD of the normalised intensity across all sections. Negative controls which were not exposed to any primary antibody showed no fluorescent staining.

\section{Data Analysis}

Data are presented as mean \pm standard error of the mean (SEM) for cellular experiments or mean \pm standard deviation (SD) for in vivo experiments. Statistical tests used to assess differences between groups are detailed in individual figure legends.

\section{Data Analysis}

All data are reported as mean values \pm s.e.m. Behavioral assays were replicated several times (3 to 10 times depending on the experiments) and averaged per animal. Statistics were then performed over the mean of animals. Statistical analysis was performed in GraphPad Prism (USA) using two-sided paired or unpaired Student's ttests, one- or two-way repeated-measures ANOVA for functional assessments, when data were distributed normally. Post hoc Tukey's or Bonferroni test was applied when appropriate. The significance level was set as $\mathrm{p}<0.05$. The nonparametric Mann-Whitney or Wilcoxon signedrank tests were used in comparisons of $<5$ rats or mice.

\section{High-speed imaging and video storage}

Mouse behaviors were recorded at $2000 \mathrm{fps}$ with a high-speed camera (Photron FastCAM Mini AX 50 170 K-M-32GB - Monochrome 170K with 32 GB memory) and attached lens (Zeiss 2/100M ZF.2mount). Mice performed behavior in rectangular plexiglass chambers on an elevated mesh platform. The camera was placed at a $\sim 45^{\circ}$ angle at $\sim 1-2$ feet away from the Plexiglas holding chambers on a tripod with geared head for Photron AX 50. CMVision IP65 infrared lights that mice cannot detect were used to adequately illuminate the paw for subsequent tracking in ProAnalyst. All data were collected on a Dell laptop computer with Photron FastCAM Analysis software (average size of video file $=\sim 2 \mathrm{~GB}$ ).

\section{Somatosensory behavior assays}

In all behavioral experiments, we used a sample size of 6-10 mice per strain, as these numbers are consistent with studies of this kind in the literature to reach statistically significant conclusions. All mice were habituated for 2 days, for one hour each day, in the Plexiglas holding chambers before testing commenced. Mice were tested in groups of five and chambers were placed in a row with barriers preventing mice from seeing each other. On testing day, mice were habituated for an additional $\sim 10$ min before stimulation and tested one at a time. Stimuli were applied through the 
mesh to the hind paw proximal to the camera. Testing only occurred when the camera's view of the paw was unobstructed. Mice received two stimuli on a given testing day ( $\mathrm{db}$ and $\mathrm{lp}$ ) and were given at least $24 \mathrm{hr}$ between each stimulus session. Stimuli were tested from least painful to most: dynamic brush then light pinprick. Dynamic brush tests were performed by wiping a concealer makeup brush (L'Oréal Paris Infallible Concealer Brush, item model number 3760228170158) across the hind paw from back to front. Light pinprick tests were performed by touching a pin (Austerlitz Insect Pins) to the hind paw of the mouse. The pin was withdrawn as soon as contact was observed.

\section{Automated paw tracking}

Proanalyst

We used ProAnalyst software to automatically track hind paw movements following stimulus application. This software allowed us to integrate automated and manually scored data, possible through the 'interpolation' feature within ProAnalyst. We were able to define specific regions of interest (paw), track, and generate data containing ' $\mathrm{x}$ ' and ' $\mathrm{y}$ ' coordinates of the paw through time. In a subset of videos, additional manual annotation was performed for increased accuracy.

\section{$\underline{\text { DeepLabCut }}$}

For deep learning-based paw tracking in DeepLabCut (DLC), we pseudo-randomly selected a subset of training frames from trials which contained the greatest behavioral variation and hand-labeled the hind paw toes, center, and heel. We trained DLC to predict toe, center, and heel positions in unlabeled video frames.

\section{Quantifying withdrawal behavior}

\section{PAWS}

Behavioral features were extracted from raw paw position time series in an automated and standardized procedure. First, the start and end of paw movement (paw at rest on the ground) were identified, and analysis was restricted to this time window. Peaks in paw height were then determined based on Savitsky-Golay smoothed estimates of paw velocity, and the first peak identified. The time of the first peak (designated $t^{*}$ ) was used to separate pre-peak behavioral feature calculations from post-peak calculations. To differentiate shaking from guarding in the post-peak period, we constructed a moving reference frame based on the principal axis of paw displacement across a sliding window (0.04 $\mathrm{s}$ in duration) for each time point, and identified periods of consecutive displacements above a specified threshold (35\% of maximum paw height) as periods of shaking. Note that in the construction of the moving reference frame the principal axes of variation were recovered via principal component analyses, which is not invariant to the sign of the recovered axes. Since displacement is measured over time it is sensitive to reversals in sign along the axis we measure it. We therefore ensured consistency by using the axis direction minimizing the angular deviation from the axis recovered at the previous time step. PAWS is open source and freely available at https://github.com/crtwomey/paws.

\section{$\underline{\mathrm{B}-\mathrm{SOiD}}$}

To determine behavioral sub-actions following foot stimulation, pose estimation data was passed along to the unsupervised behavioral discovery and extraction algorithm, B-SOiD. Experimentalists processing this data were blind to the experimental condition. Position coordinates of fore paw hind paw motion tracked by DeepLabCut in samples of 2000fps video, and then were imported to the BSOiD app to identify unique behavioral clusters in response to pain stimulus. Data from dynamic brush and light prick Hour-4 sessions were combined to develop a generalized B-SOiD model of pain response. The frame rate was scaled down to $1 / 7$ th of the original to help B-SOiD extract sub- 
behavioral features. Data used in the B-SOiD model were the hind paw toe, hind paw center-paw and two static reference positions. The two static reference points were the initial positions of the fore paw the maximum elevation of toe post stimulation. B-SOiD performed nonlinear embedding to transform 16-dimensional data to 5dimensional UMAP space. The 16-dimensional data include frame by frame calculation of distance and angle between all four points as well as the speed of the two body parts. 11 behavioral clusters were identified and used to train the random forest classifier of the algorithm, which was then used to assign behavioral labels to data from all epochs and stimulation types. Every frame was labeled, and a smoothing kernel was used to eliminate any subactions lasting under 2.5 seconds (5 frames). Importantly, beyond the spatiotemporal relationship values between the points, no other information was available to the algorithm. BSOiD is open source and freely available at https://github.com/YttriLab/B-SOID.

\section{Acknowledgements}

LAP and EStJS acknowledge the work of University of Cambridge Combined Animal Facility staff. LAP was supported by the University of Cambridge BBSRC Doctoral Training Programme (BB/M011194/1). EStJS acknowledges support from Versus Arthritis (RG 21973). IAS, WF, HR, JA, ZAJ were supported from startup funds from the University of Pennsylvania and Columbia University, a grant from the National Institutes of Health NIH/NIDCR, R00-DE026807, the Rita Allen Foundation, and the Alfred P. Sloan Foundation. VEA, MAT, MB are supported by startup funds from Rutgers University. VEA and MB are also supported by the Pew Charitable Trust. MAT is also supported by Tourette Association of America, New Jersey Center for Tourette Syndrome, and a Robert Wood Johnson foundation grant.

\section{Author Contributions}

LAP and MB contributed equally to the work. EStJS, IAS, and VEA conceived the research study. EStJS, IAS, EY, and VEA designed experiments. LAP performed the electrophysiology experiments. MB, HR, ZAJ, and WF performed the behavioral experiments. ZAJ, WF, JA, NM, and EY performed the analysis for PAWS and B-SOiD. MB, MAT, JKT, MR performed the analysis for Motion Sequencing. LAP, MB, IAS, HR, ZAJ, WF, EStJS, MR, and VEA wrote the manuscript. All authors contributed to the editing of the final document.

\section{Inclusion and Diversity Statement}

All the authors in this study actively support inclusion, diversity, and equality in science. The two senior authors, and several co-authors are from racial and ethnic groups underrepresented in science. Gender and sexual orientation diversity is also highlighted in the composition of this team, including representation of women as the first and last author. One or more of the authors of this paper received support from a program designed to increase minority representation in science, including the PDEP fellowship from Burroughs Wellcome Fund. While citing references scientifically relevant for this work, we also actively worked to promote gender balance in our reference list.

\section{Competing Interests}

The authors declare no conflict of interest with any of the data presented in this article.

\section{References}

Abdus-Saboor, I., Fried, N.T., Lay, M., Burdge, J., Swanson, K., Fischer, R., Jones, J., Dong, P., Cai, W., Guo, X., et al. (2019). Development of a Mouse Pain Scale Using Sub-second Behavioral Mapping and Statistical Modeling. Cell Rep 28, 16231634.e1624. 
Aoki, T., and Narumiya, S. (2012). Prostaglandins and chronic inflammation. Trends Pharmacol. Sci. 33, 304-311.

Barik, A., Thompson, J.H., Seltzer, M., Ghitani, N., and Chesler, A.T. (2018). A Brainstem-Spinal Circuit Controlling Nocifensive Behavior. Neuron 100, 1491-1503.e1493.

Barrot, M. (2012). Tests and models of nociception and pain in rodents. Neuroscience 211, 39-50.

Buisseret, B., Guillemot-Legris, O., Muccioli, G.G., and Alhouayek, M. (2019). Prostaglandin D2glycerol ester decreases carrageenan-induced inflammation and hyperalgesia in mice. Biochim Biophys Acta Mol Cell Biol Lipids 1864, 609-618.

Busch, U., Schmid, J., Heinzel, G., Schmaus, H., Baierl, J., Huber, C., and Roth, W. (1998). Pharmacokinetics of meloxicam in animals and the relevance to humans. Drug Metab Dispos 26, 576584.

Chakrabarti, S., Pattison, L.A., Singhal, K., Hockley, J.R.F., Callejo, G., and Smith, E.S.J. (2018). Acute inflammation sensitizes kneeinnervating sensory neurons and decreases mouse digging behavior in a TRPV1-dependent manner. Neuropharmacology 143, 49-62.

Choi, S., Hachisuka, J., Brett, M.A., Magee, A.R., Omori, Y., Iqbal, N.-U.-A., Zhang, D., DeLisle, M.M., Wolfson, R.L., Bai, L., et al. (2020). Parallel ascending spinal pathways for affective touch and pain. Nature 587, 258-263.

Corder, G., Ahanonu, B., Grewe, B.F., Wang, D., Schnitzer, M.J., and Scherrer, G. (2019). An amygdalar neural ensemble that encodes the unpleasantness of pain. Science 363, 276-281.

da Silva Serra, I., Husson, Z., Bartlett, J.D., and Smith, E.S.J. (2016). Characterization of cutaneous and articular sensory neurons. Mol Pain 12, 174480691663638.

Davidson, S., Copits, B.A., Zhang, J., Page, G., Ghetti, A., and Gereau, R.W. (2014). Human sensory neurons: Membrane properties and sensitization by inflammatory mediators. Pain 155 , 1861-1870.

Ghouri, A., and Conaghan, P.G. (2019). Treating osteoarthritis pain: recent approaches using pharmacological therapies. Clin Exp Rheumatol 37 Suppl 120, 124-129.

Gold, M.S., Reichling, D.B., Shuster, M.J., and Levine, J.D. (1996). Hyperalgesic agents increase a tetrodotoxin-resistant $\mathrm{Na}+$ current in nociceptors. Proc. Natl. Acad. Sci. U.S.a. 93, 1108-1112.

Hargreaves, K., Dubner, R., Brown, F., Flores, C., and Joris, J. (1988). A new and sensitive method for measuring thermal nociception in cutaneous hyperalgesia. Pain 32, 77-88.

Hsu, A.I., and Yttri, E.A. (2021). An Open Source Unsupervised Algorithm for Identification and Fast Prediction of Behaviors. bioRxiv

Hua, T., Chen, B., Lu, D., Sakurai, K., Zhao, S., Han, B.-X., Kim, J., Yin, L., Chen, Y., Lu, J., et al. (2020). General anesthetics activate a potent central pain-suppression circuit in the amygdala. Nat. Neurosci. 23, 854-868.

Huang, T., Lin, S.-H., Malewicz, N.M., Zhang, Y., Zhang, Y., Goulding, M., LaMotte, R.H., and Ma, Q. (2019). Identifying the pathways required for coping behaviours associated with sustained pain. Nature 565, 86-90.

Ibuki, T., Matsumura, K., Yamazaki, Y., Nozaki, T., Tanaka, Y., and Kobayashi, S. (2003). Cyclooxygenase-2 is induced in the endothelial cells throughout the central nervous system during carrageenan-induced hind paw inflammation; its possible role in hyperalgesia. Journal of Neurochemistry $86,318-328$.

Jones, J.M., Foster, W., Twomey, C.R., Burdge, J., Ahmed, O.M., Pereira, T.D., Wojick, J.A., Corder, G., Plotkin, J.B., and Abdus-Saboor, I. (2020). A machine-vision approach for automated pain measurement at millisecond timescales. Elife 9, 1623 . 
Le, Q., and Mikolov, T. (2014). Distributed Representations of Sentences and Documents. E.P. Xing, and T. Jebara, eds. (Bejing, China: PMLR), pp. $1188-1196$.

Mao, J. (2012). Current challenges in translational pain research. Trends Pharmacol. Sci. 33, 568-573.

Miyake, S., Higuchi, H., Honda-Wakasugi, Y., Fujimoto, M., Kawai, H., Nagatsuka, H., Maeda, S., and Miyawaki, T. (2019). Locally injected ivabradine inhibits carrageenan-induced pain and inflammatory responses via hyperpolarizationactivated cyclic nucleotide-gated (HCN) channels. PLoS ONE 14, e0217209.

Moilanen, L.J., Laavola, M., Kukkonen, M., Korhonen, R., Leppänen, T., Högestätt, E.D., Zygmunt, P.M., Nieminen, R.M., and Moilanen, E. (2012). TRPA1 contributes to the acute inflammatory response and mediates carrageenaninduced paw edema in the mouse. Sci Rep 2, 380386.

Morris, C.J. (2003). Carrageenan-induced paw edema in the rat and mouse. Methods Mol Biol 225, 115-121.

Nassar, M.A., Stirling, L.C., Forlani, G., Baker, M.D., Matthews, E.A., Dickenson, A.H., and Wood, J.N. (2004). Nociceptor-specific gene deletion reveals a major role for Nav1.7 (PN1) in acute and inflammatory pain. Proc. Natl. Acad. Sci. U.S.a. 101, 12706-12711.

Ott, S., and Maihöfner, C. (2018). Signs and Symptoms in 1,043 Patients with Complex Regional Pain Syndrome. J Pain 19, 599-611.

Pritchard, R.A., Falk, L., Larsson, M., Leinders, M., and Sorkin, L.S. (2016). Different phosphoinositide 3-kinase isoforms mediate carrageenan nociception and inflammation. Pain $157,137-146$.

Rehurek, R., and Sojka, P. (2010). Software Framework for Topic Modelling with Large Corpora. In Proceedings of LREC Workshop New Challenges for NLP Frameworks, (Valletta, Malta), pp. pp.46-pp.50.
Rock, E.M., Limebeer, C.L., and Parker, L.A. (2018). Effect of cannabidiolic acid and $\Delta 9$ tetrahydrocannabinol on carrageenan-induced hyperalgesia and edema in a rodent model of inflammatory pain. Psychopharmacology (Berl) 235, 3259-3271.

Smith, E.S., and Lewin, G.R. (2009). Nociceptors: a phylogenetic view. J Comp Physiol A Neuroethol Sens Neural Behav Physiol 195, 1089-1106.

Watanabe, M., Ueda, T., Shibata, Y., Kumamoto, N., and Ugawa, S. (2015). The role of TRPV1 channels in carrageenan-induced mechanical hyperalgesia in mice. Neuroreport 26, 173-178.

Wiltschko, A.B., Johnson, M.J., Iurilli, G., Peterson, R.E., Katon, J.M., Pashkovski, S.L., Abraira, V.E., Adams, R.P., and Datta, S.R. (2015). Mapping Sub-Second Structure in Mouse Behavior. Neuron 88, 1121-1135.

Wiltschko, A.B., Tsukahara, T., Zeine, A., Anyoha, R., Gillis, W.F., Markowitz, J.E., Peterson, R.E., Katon, J., Johnson, M.J., and Datta, S.R. (2020). Revealing the structure of pharmacobehavioral space through motion sequencing. Nat. Neurosci. 23, 1433-1443.

Woodcock, J. (2009). A difficult balance--pain management, drug safety, and the FDA. N. Engl. J. Med. 361, 2105-2107. 